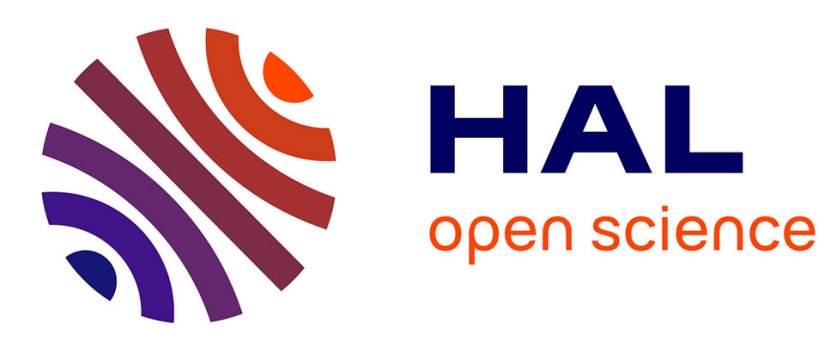

\title{
Hinfinity control under asymptotic tracking constraint and nonproper output weights
}

\author{
Mohamed Yagoubi, Yu Feng, Yanyu Huang
}

\section{To cite this version:}

Mohamed Yagoubi, Yu Feng, Yanyu Huang. Hinfinity control under asymptotic tracking constraint and nonproper output weights. International Journal of Control, 2019, 10.1080/00207179.2018.1562223 . hal-01958326

\section{HAL Id: hal-01958326 \\ https://hal.science/hal-01958326}

Submitted on 11 Feb 2022

HAL is a multi-disciplinary open access archive for the deposit and dissemination of scientific research documents, whether they are published or not. The documents may come from teaching and research institutions in France or abroad, or from public or private research centers.
L'archive ouverte pluridisciplinaire HAL, est destinée au dépôt et à la diffusion de documents scientifiques de niveau recherche, publiés ou non, émanant des établissements d'enseignement et de recherche français ou étrangers, des laboratoires publics ou privés. 


\title{
$\mathcal{H}_{\infty}$ control under asymptotic tracking constraint and nonproper output weights
}

\author{
Yu Feng ${ }^{\mathrm{a}}$, Yanyu Huang ${ }^{\mathrm{a}}$, and Mohamed Yagoubi ${ }^{\mathrm{b}}$ \\ anformation Engineering College, Zhejiang University of Technology, 288 Liuhe Road, \\ Hangzhou, 310032, P.R. China; ${ }^{\mathrm{b}}$ IMT Atlantique-LS2N Nantes, La Chantrerie, 4 rue Alfred \\ Kastler, 44307 Nantes, France
}

\section{ARTICLE HISTORY}

Compiled January 2, 2018

\begin{abstract}
This paper presents a complete solution to the measurement output feedback $\mathcal{H}_{\infty}$ control problem subject to asymptotic tracking of an infinite energy reference and the presence of nonproper output weights. Relying on generalized Sylvester-type equations, a set of all stabilizing controllers is characterized, explicitly exhibiting the impacts of the reference signal and the output weight on the resulting controllers. Based on this specific parameterization, additional disturbance attenuation is tackled, and both full-order and reduced-order controller synthese are discussed. It is observed that the multiobjective problem is equivalent to a standard problem for an axillary system constructed from the original weighted system with the control input matrix and measurement output matrix being augmented by solutions to generalized Sylvester-type equations. An example is included to show the effectiveness of the present results.
\end{abstract}

\section{KEYWORDS}

Asymptotic tracking; controller parameterization; $\mathcal{H}_{\infty}$ performance; nonproper weights; reduced-order controller; robust control

\section{Introduction}

One of the fundamental issues in systems and control theory is to track (reject) infinite energy references (disturbances) asymptotically, which is also referred to as the regulation problem Stoorvogel, Saberi, and Sannuti (2000). Such a problem commonly appears in practical engineering systems, such as helicopters Cai, Chen, Peng, Lee, and Dong (2008), disk drive servo system Lan, Thum, and Chen (2010), lateral vehicle control Fauvel, Claveau, and Chevrel (2013), spacecraft Fadakar, Fidan, and Huissoon (2017), robotic manipulator Andreev and Peregudova (2017), etc. Various solutions have been provided to guarantee tracking performance ; for example, repetitive control Pipeleers, Demeulenaere, Schutter, and Swevers (2008); Tomizuka (2008), adaptive control Zhang and Xie (2014), and nonlinear control Chen, Lee, Peng, and Venkataramanan (2003); Feng and Ho (2014). Among these different techniques, one seminal result, known as the internal model principle Francis (1977); Wonham (1985), achieves exact asymptotic tracking by a structured controller containing a copy of 
the dynamics of the so-called exo-system. Extensions of this scheme have been widely developed by considering other performance objectives and such multiobjective problems have been extensively investigated in the literature; e.g., see Köroğlu and Scherer (2011); Meng, Yang, and Dimarogonas (2015); Su and Huang (2014); Wieland, Sepulchre, and Allgöwer (2011); Yu, Zhao, and Wu (2014) and the references therein.

An alternative approach to tackling the aforementioned problems is the use of weighting filters Feng and Yagoubi (2016); Meinsma (1995); Mita, Xin, and Anderson (2000). It is well known that, in many problems, weights are often required for the definition of a standard model consisting of a physical plant, disturbances and reference signals, and control objectives. A well recognized example of adopting weights is the $\mathcal{H}_{\infty}$ control in which it is often desirable to choose a weight with a pole at the origin since the closed-loop system is finite only if the sensitivity has a zero at the origin. This choice indicates that the underlying stabilizing controller achieves either the perfect rejection of constant disturbances or the tracking of constant references. Moreover, in order to avoid undesirable high-frequency noise sensitivity and limited robustness, it is also often advisable to select a nonproper weight, whose $\mathcal{H}_{\infty}$ norm is generally outside the desirable closed-loop bandwidth Meinsma (1995). However, the use of weights potentially introduces some unstabilizable or undetectable finite dynamics, even uncontrollable or unobservable impulsive elements, if nonproper weights are involved, into the standard model. Under these circumstances, the stabilization issue yields a nonstandard problem which traditional methods fail to solve, since the conventional internal stability can not usually be achieved. These undesirable elements can be treated approximately ; for example, by slight perturbation to render the problem standard Chiang and Safonov (1992). However, this approach is vulnerable to the issues related to lightly-damped poles and may lead to higher order and not strictly proper controllers. To solve such problems, Kwakernaak Kwakernaak (1993) uses the so-called polynomial method, which copes naturally with nonproper weights, but can not be applied to the case in which weights have imaginary poles. Plant augmentation is rather similar to the "plant state tapping" technique which is also used to address unstable and nonproper weights Meinsma (1995). Recently, through the framework of descriptor systems Dai (1989); Feng and Yagoubi (2017); Xu and Lam (2006), which can easily model nonproper elements, the concept of comprehensive admissibility Feng, Yagoubi, and Chevrel (2012a, 2012b), which expands the results in Liu, Zhang, and Mita (1997); Mita et al. (2000) into the nonproper case, has been introduced to handle such nonstandard problems, and $\mathcal{H}_{2}$ and $\mathcal{H}_{\infty}$ performance control are also solved via Riccati-type solutions.

In this paper, we consider the measurement output feedback $\mathcal{H}_{\infty}$ control with exact asymptotic tracking constraint and nonproper output weighting filter, and we present a complete solution to such a multiobjective problem. The infinite energy reference to be tracked is described as an autonomous weight in order to address the problem within a uniform framework. A set of all stabilizing controllers is first explicitly characterized in terms of weights' dynamics and solutions to generalized Sylvester-type equations. Based on the structured controller, the nonstandard problem is transformed into a standard control problem with respect to an auxiliary system, which is constructed from the original weighted system with the control input matrix and measurement output matrix being augmented by solutions to generalized Sylvester-type equations, and additional $\mathcal{H}_{\infty}$ performance is further tackled through LMI-based solutions. Moreover, for the sake of easy implementation, reduced-order $\mathcal{H}_{\infty}$ controller synthesis is included. Unlike the Riccati-type solution reported in Feng et al. (2012a, 2012b), the current results do not require assumptions of invariant zeros, and the $\mathcal{H}_{\infty}$ performance design 
is independent of solutions to generalized Sylvester-type equations, which implies a convex optimization. The remainder of this paper is organized as follows. The multiobjective problem under consideration is defined in Section 2. Section 3 presents the parameterization of all stabilizing controllers under asymptotic tracking and potentially nonproper output weights. Based on this specific structure, both full-order and reduced-order $\mathcal{H}_{\infty}$ controller design are further discussed in Section 4 . An example is included to illustrate the effectiveness of the present results in Section 5, and concluding remarks are given in Section 6. Some fundamental concepts of descriptor systems are recalled in Appendix A.

Notation: The notation used in this paper is fairly standard. For a real square matrix $X$ the notation $X \geq 0$ (respectively $X>0$ ) means that the matrix $X$ is symmetric and positive-semi-definite (respectively positive definite). The superscripts ' $T$ 'and ' -1 ' represent the transpose and inverse, respectively. $\operatorname{Tr}(\cdot)$ and $\lambda(\cdot)$ are the trace and eigenvalues of the matrix, while the notation $\mathbf{H e}\{P\}$ stands for $P^{\top}+P$. For a symmetric matrix represented blockwise, off-diagonal blocks are abbreviated by ' $\bullet$ '. $\mathcal{F}_{l}(\cdot, \cdot)$ stands for the lower linear fractional transformation. Moreover, for a matrix $\Lambda \in \mathbb{R}^{m \times n}$ with $m>n$, let $\Lambda^{\perp} \in \mathbb{R}^{(m-n) \times m}$ be any matrix satisfying $\Lambda^{\perp} \Lambda=0$ and $\Lambda^{\perp}\left(\Lambda^{\perp}\right)^{\top}>0$. Note that $\Lambda^{\perp}$ exists if and only if $\Lambda$ has linearly dependent rows. For $m<n$, let $\Lambda^{\perp} \triangleq\left(\left(\Lambda^{\top}\right)^{\perp}\right)^{\top}$. For simplicity, the arguments of a function will sometimes be omitted when no confusion can arise. Besides, unless stated otherwise, all matrices used in this paper are assumed to have appropriate dimensions.

\section{Problem formulation}

The problem setup is depicted in Fig. 1, where $\tilde{G}$ stands for the physical plant described by the following continuous-time linear time-invariant (LTI) system

$$
\tilde{G}:\left\{\begin{aligned}
\dot{x} & =A_{g} x+B_{r} r+B_{w} w+B_{u} u, \\
v & =C_{v} x+D_{v r} r+D_{v w} w+D_{v u} u, \\
y & =C_{g} x+D_{r} r+D_{w} w \\
e & =C_{e} x+D_{e r} r+D_{e w} w+D_{e u} u,
\end{aligned}\right.
$$

where $x \in \mathbb{R}^{n}, y \in \mathbb{R}^{p}, v \in \mathbb{R}^{l}, e \in \mathbb{R}^{q}, r \in \mathbb{R}^{p_{i}}, w \in \mathbb{R}^{w}, z \in \mathbb{R}^{p_{o}}$ and $u \in \mathbb{R}^{m}$ are the plant state, measurement output, controlled output, tracking error, exogenous reference, external disturbance, weighted output and control input, respectively. The matrices $A_{g} \in \mathbb{R}^{n \times n}, B_{r} \in \mathbb{R}^{n \times p_{i}}, B_{w} \in \mathbb{R}^{n \times w}, B_{u} \in \mathbb{R}^{n \times m}, C_{v} \in \mathbb{R}^{l \times n}, C_{g} \in \mathbb{R}^{p \times n}$, $C_{e} \in \mathbb{R}^{q \times n}, D_{v r} \in \mathbb{R}^{l \times p_{i}}, D_{v w} \in \mathbb{R}^{l \times w}, D_{v u} \in \mathbb{R}^{l \times m}, D_{r} \in \mathbb{R}^{p \times p_{i}}, D_{w} \in \mathbb{R}^{p \times w}$, $D_{\text {er }} \in \mathbb{R}^{q \times p_{i}}, D_{\text {ew }} \in \mathbb{R}^{q \times w}$, and $D_{\text {eu }} \in \mathbb{R}^{q \times m}$ are known constant matrices. The direct feedthrough matrix from $u$ to $y$ is left out to make the arguments simpler and can be handled via standard methods.

The exogenous reference $r$ to follow is assumed to be generated by the linear autonomous system $W_{i}$, known as the exo-system,

$$
W_{i}:\left\{\begin{aligned}
\dot{x}_{i} & =A_{i} x_{i} \\
r & =C_{i} x_{i}
\end{aligned}\right.
$$

with $A_{i} \in \mathbb{R}^{n_{i} \times n_{i}}$ and $C_{i} \in \mathbb{R}^{p_{i} \times n_{i}}$. The weighted output $z$ is related to the controlled 


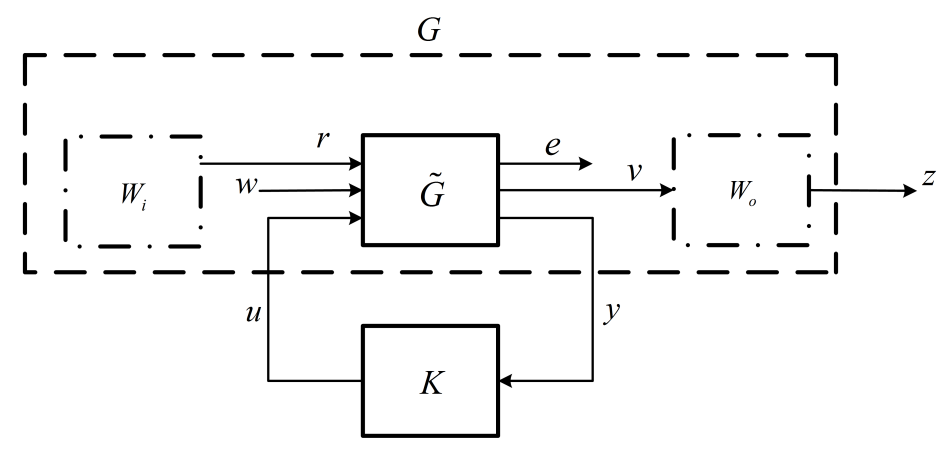

Figure 1. Problem setup

output $v$ by the filter $W_{o}$ of the form

$$
W_{o}:\left\{\begin{aligned}
E_{o} \dot{x}_{o} & =A_{o} x_{o}+B_{o} v, \\
z & =C_{o} x_{o}+D_{o} v,
\end{aligned}\right.
$$

where $E_{o} \in \mathbb{R}^{n_{o} \times n_{o}}, A_{o} \in \mathbb{R}^{n_{o} \times n_{o}}, B_{o} \in \mathbb{R}^{n_{o} \times l}, C_{o} \in \mathbb{R}^{p_{o} \times n_{o}}$, and $D_{o} \in \mathbb{R}^{p_{o} \times l}$ are known real constant matrices. The matrix $E_{o}$ may be singular, i.e. $\operatorname{rank}\left(E_{o}\right)=r_{o} \leq n_{o}$. Note that systems of the form (3) are called descriptor (singular) systems and are known for their capacity to preserve the structure of physical systems and to describe nondynamic constraints and impulsive behaviors Dai (1989); Feng and Yagoubi (2017); Xu and Lam (2006). The purpose of using descriptor representation here is to introduce nonproper output weights. Since the descriptor system itself is not the main focus of this paper, we do not give too much detail here, but include some basic notations in Appendix A to be self-contained.

The generalized system, incorporating the exo-system $W_{i}$, output weight $W_{o}$ and physical plant $\tilde{G}$, is thus written by

$$
G:\left\{\begin{array}{rl}
E \dot{\zeta} & =A \zeta+B_{1} w+B_{2} u, \\
z & =C_{1} \zeta+D_{11} w+D_{12} u, \\
y & =C_{2} \zeta+D_{21} w, \\
e & =C_{3} \zeta+D_{31} w+D_{22} u,
\end{array}:=\left[\begin{array}{c|cc}
A-s E & B_{1} & B_{2} \\
\hline C_{1} & D_{11} & D_{12} \\
C_{2} & D_{21} & 0 \\
C_{3} & D_{31} & D_{32}
\end{array}\right]\right.
$$

where

$$
\begin{aligned}
& \zeta=\left[\begin{array}{c}
x_{o} \\
x \\
x_{i}
\end{array}\right], \quad E=\left[\begin{array}{ccc}
E_{o} & 0 & 0 \\
0 & I_{n} & 0 \\
0 & 0 & I_{n_{i}}
\end{array}\right], \quad A=\left[\begin{array}{ccc}
A_{o} & B_{o} C_{v} & B_{o} D_{v r} C_{i} \\
0 & A_{g} & B_{r} C_{i} \\
0 & 0 & A_{i}
\end{array}\right], \\
& B_{1}=\left[\begin{array}{c}
B_{o} D_{v w} \\
B_{w} \\
0
\end{array}\right], \quad B_{2}=\left[\begin{array}{c}
B_{o} D_{v u} \\
B_{u} \\
0
\end{array}\right], \quad C_{1}=\left[\begin{array}{lll}
C_{o} & D_{o} C_{v} & D_{o} D_{v r} C_{i}
\end{array}\right] \text {, } \\
& C_{2}=\left[\begin{array}{lll}
0 & C_{g} & D_{r} C_{i}
\end{array}\right], \quad C_{3}=\left[\begin{array}{lll}
0 & C_{e} & D_{e r} C_{i}
\end{array}\right], \quad D_{11}=D_{o} D_{v w}, \\
& D_{12}=D_{o} D_{v u}, \quad D_{21}=D_{w}, \quad D_{31}=D_{e w}, \quad D_{32}=D_{e u} .
\end{aligned}
$$


The measurement feedback controller $K$ is assumed to be

$$
K:=\left[\begin{array}{c|c}
A_{K}-s E_{K} & B_{K} \\
\hline C_{K} & D_{K}
\end{array}\right],
$$

where $E_{K} \in \mathbb{R}^{n_{k} \times n_{k}}, A_{K} \in \mathbb{R}^{n_{k} \times n_{k}}, B_{K} \in \mathbb{R}^{n_{k} \times p}, C_{K} \in \mathbb{R}^{m \times n_{k}}$ and $D_{K} \in \mathbb{R}^{m \times p}$. The matrix $E_{K}$ may be singular, i.e. $\operatorname{rank}\left(E_{K}\right)=r_{k} \leq n_{k}$.

The closed-loop system formed by $G$ and $K$ is denoted by $\mathcal{F}_{l}(G, K)$, and the transform function from the external disturbance $w$ to weighted output $z$ of $\mathcal{F}_{l}(G, K)$ is denoted by $T_{z w}(s)$.

Problem 2.1 (Asymptotic Tracking with Weighted $\mathcal{H}_{\infty}$ Performance). The problem under consideration is to find a measurement feedback controller such that the following conditions are satisfied

(C1) (Asymptotic tracking) The tracking error $e(t)$ satisfies $\lim _{t \rightarrow \infty} e(t)=0$ for any $w(t) \in \mathcal{L}_{2}$, and for all $x(0) \in \mathbb{R}^{n}$, and $x_{i}(0) \in \mathbb{R}^{n_{i}}$

(C2) $\left(\mathcal{H}_{\infty}\right.$ performance) The $\mathcal{H}_{\infty}$ norm of $T_{z w}(s)$ is bounded by $\gamma$ for some $\gamma>0$.

In order to solve this problem, we introduce here two partitions of $G$ with regard to $W_{i}$ and $W_{o}$, denoted as $G^{W_{i}}$ and $G^{W_{o}}$, respectively,

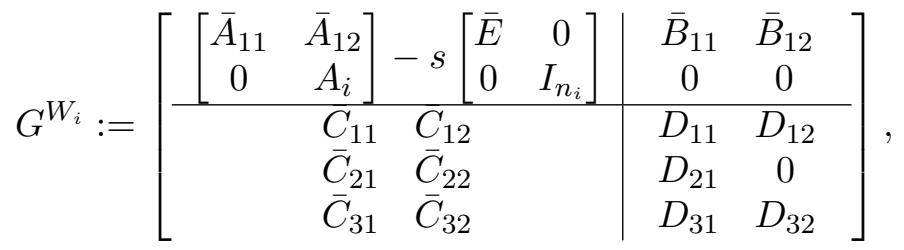

where

$$
\begin{aligned}
& \bar{E}=\left[\begin{array}{cc}
E_{o} & 0 \\
0 & I_{n}
\end{array}\right], \quad \bar{A}_{11}=\left[\begin{array}{cc}
A_{o} & B_{o} C_{v} \\
0 & A_{g}
\end{array}\right], \quad \bar{A}_{12}=\left[\begin{array}{c}
B_{o} D_{v r} C_{i} \\
B_{r} C_{i}
\end{array}\right], \\
& \bar{B}_{11}=\left[\begin{array}{c}
B_{o} D_{v r} \\
B_{w}
\end{array}\right], \quad \bar{B}_{12}=\left[\begin{array}{cc}
B_{o} D_{v u} \\
B_{u}
\end{array}\right], \quad \bar{C}_{11}=\left[\begin{array}{ll}
C_{o} & D_{o} C_{v}
\end{array}\right], \\
& \bar{C}_{12}=D_{o} D_{v r} C_{i}, \quad \bar{C}_{21}=\left[\begin{array}{ll}
0 & C_{g}
\end{array}\right], \quad \bar{C}_{22}=D_{r} C_{i}, \quad \bar{C}_{31}=\left[\begin{array}{ll}
0 & C_{e}
\end{array}\right], \\
& \bar{C}_{32}=D_{e r} C_{i}, \quad D_{11}=D_{o} D_{v w}, \quad D_{12}=D_{o} D_{v u}, \quad D_{21}=D_{w}, \\
& D_{31}=D_{e w}, \quad D_{32}=D_{e u} ;
\end{aligned}
$$

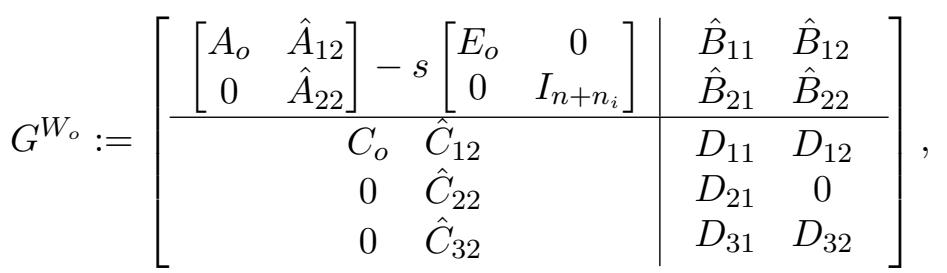


where

$$
\begin{aligned}
& \hat{A}_{12}=\left[\begin{array}{ll}
B_{o} C_{v} & B_{o} D_{v r} C_{i}
\end{array}\right], \quad \hat{A}_{22}=\left[\begin{array}{cc}
A_{g} & B_{r} C_{i} \\
0 & A_{i}
\end{array}\right], \quad \hat{B}_{11}=B_{o} D_{v w}, \\
& \hat{B}_{12}=B_{o} D_{v u}, \quad \hat{B}_{21}=\left[\begin{array}{c}
B_{w} \\
0
\end{array}\right], \quad \hat{B}_{22}=\left[\begin{array}{c}
B_{u} \\
0
\end{array}\right], \quad \hat{C}_{12}=\left[\begin{array}{ll}
D_{o} C_{v} & D_{o} D_{v r} C_{i}
\end{array}\right], \\
& \hat{C}_{22}=\left[\begin{array}{ll}
C_{g} & D_{r} C_{i}
\end{array}\right], \quad \hat{C}_{32}=\left[\begin{array}{ll}
C_{e} & D_{e r} C_{i}
\end{array}\right] .
\end{aligned}
$$

Before ending this section, the following assumptions are made.

(A1) $W_{i}$ and $W_{o}$ only possess unstable and impulsive modes;

(A2) $\left(\bar{E}, \bar{A}_{11}, \bar{B}_{12}\right)$ is finite dynamics stabilizable and impulse controllable;

(A3) $\left(\hat{A}_{22}, \hat{C}_{22}\right)$ is detectable.

Assumption (A1) is made without loss of generality to simplify the argument, since stable modes, if they exist, of $W_{i}$ and $W_{o}$ do not affect the internal stability of the underlying closed-loop system and can always be incorporated into the physical plant $\tilde{G}$. Assumptions (A2) and (A3) are necessary for the existence of a stabilizing measurement feedback controller to the weighted system $G$. Moreover, it is worth noting that due to the use of unstable and nonproper weights, the multiobjective problem under consideration results in a design problem with unstabilizable and undetectable dynamics, which standard methods cannot solve. In this paper, we present a complete solution to this nonstandard multiobjective problem through a structured controller together with LMI-based conditions to both full-order and reduced-order controller synthesis.

\section{Controller paramaterization}

In this section, we show a specific parameterization for stabilizing controller design to fulfil the asymptotic tracking and stability of the transfer function $T_{z w}(s)$. To this end, we mildly modify Condition (C2) in Problem 2.1 to

(C'2) The transfer function $T_{z w}(s)$ is stable.

The following theorem gives the necessary and sufficient solution to Conditions (C1) and (C'2).

Theorem 3.1. Consider the partitions (6) and (7). Conditions $(\boldsymbol{C} 1)$ and $\left(\boldsymbol{C}^{\prime}\right.$ '2) are satisfied, if and only if there exist matrices $X_{i} \in \mathbb{R}^{\left(n+n_{o}\right) \times n_{i}}, Y_{i} \in \mathbb{R}^{\left(n+n_{o}\right) \times n_{i}}$, $\Pi_{i} \in \mathbb{R}^{m \times n_{i}}, X_{o} \in \mathbb{R}^{n_{o} \times\left(n+n_{i}\right)}, Y_{o} \in \mathbb{R}^{n_{o} \times\left(n+n_{i}\right)}$ and $\Pi_{o} \in \mathbb{R}^{n_{o} \times p}$ such that the following generalized Sylvester-type equations hold.

$$
\begin{aligned}
& \left\{\begin{aligned}
\bar{A}_{12} & =\bar{A}_{11} Y_{i}+\bar{B}_{12} \Pi_{i}+X_{i} A_{i}, \\
\bar{C}_{32} & =\bar{C}_{31} Y_{i}+D_{32} \Pi_{i}, \\
0 & =\bar{E} Y_{i}+X_{i} .
\end{aligned}\right. \\
& \left\{\begin{aligned}
\hat{A}_{12} & =\Pi_{o} \hat{C}_{22}-X_{o} \hat{A}_{22}+A_{o} Y_{o}, \\
\hat{B}_{11} & =\Pi_{o} D_{21}-X_{o} \hat{B}_{21}, \\
0 & =E_{o} Y_{o}-X_{o} .
\end{aligned}\right.
\end{aligned}
$$

Moreover, the measurement feedback controller $K$ is illustrated in Fig. 2 and 


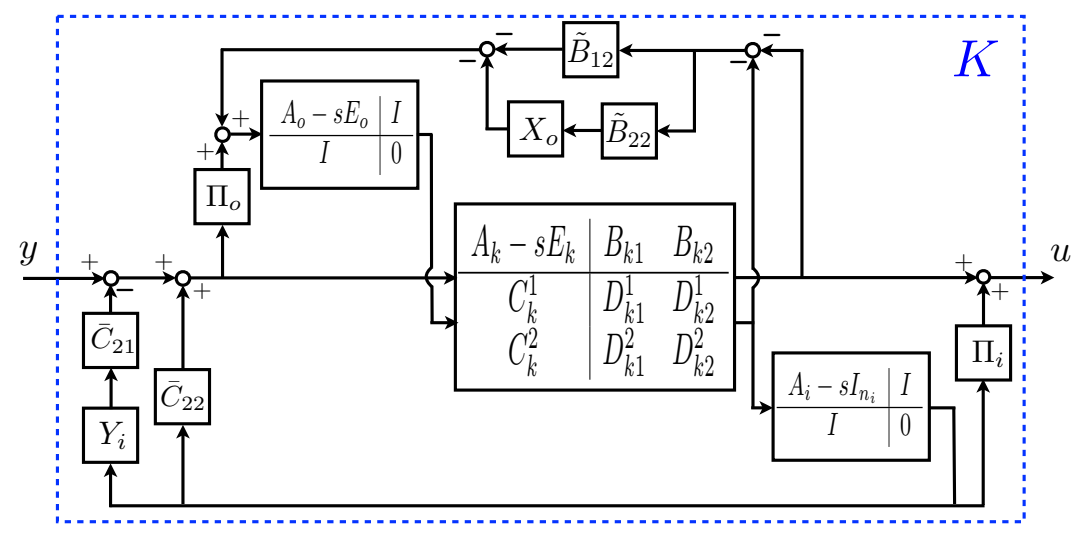

Figure 2. Structured controller

parametrized by

$$
K:=\left[\begin{array}{c|c}
A_{K}-s E_{K} & B_{K} \\
\hline C_{K} & D_{k 1}^{1}
\end{array}\right],
$$

where

$$
\begin{aligned}
& E_{K}=\left[\begin{array}{ccc}
I_{n_{i}} & 0 & 0 \\
0 & E_{k} & 0 \\
0 & 0 & E_{o}
\end{array}\right], \quad A_{K}=\left[\begin{array}{ccc}
A_{i}+D_{k 1}^{2} \Gamma_{i} & C_{k}^{2} & D_{k 2}^{2} \\
B_{k 1} \Gamma_{i} & A_{k} & B_{k 2} \\
\Omega_{o} \Gamma_{i} & -\Gamma_{o} C_{k} & A_{o}-\Gamma_{o} D_{k 2}
\end{array}\right] \\
& B_{K}=\left[\begin{array}{c}
D_{k 1}^{2} \\
B_{k 1} \\
\Omega_{o}
\end{array}\right], \quad C_{K}=\left[\begin{array}{lll}
\Omega_{i} & C_{k}^{1} & D_{k 2}^{1}
\end{array}\right], \quad \Gamma_{i}=\bar{C}_{22}-\bar{C}_{21} Y_{i}, \\
& \Gamma_{o}=-X_{o} \tilde{B}_{22}-\tilde{B}_{12}, \quad \tilde{B}_{12}=\left[\begin{array}{ll}
\hat{B}_{12} & X_{i}^{1}
\end{array}\right], \quad \tilde{B}_{22}=\left[\begin{array}{c|c}
\hat{B}_{22} & X_{i}^{2} \\
I_{n_{i}}
\end{array}\right], \\
& \Omega_{i}=D_{k 1}^{1} \Gamma_{i}+\Pi_{i}, \quad \Omega_{o}=\Pi_{o}-\Gamma_{o} D_{k 1}, \quad X_{i}^{1}=\left[\begin{array}{ll}
I_{n_{o}} & 0
\end{array}\right] X_{i}, \\
& X_{i}^{2}=\left[\begin{array}{ll}
0 & I_{n}
\end{array}\right] X_{i}, \quad C_{k}=\left[\begin{array}{c}
C_{k}^{1} \\
C_{k}^{2}
\end{array}\right], \quad D_{k 1}=\left[\begin{array}{c}
D_{k 1}^{1} \\
D_{k 1}^{2}
\end{array}\right], \quad D_{k 2}=\left[\begin{array}{c}
D_{k 2}^{1} \\
D_{k 2}^{2}
\end{array}\right],
\end{aligned}
$$

and the matrices $E_{k}, A_{k}, B_{k 1}, B_{k 2}, C_{k}^{1}, C_{k}^{2}, D_{k 1}^{1}, D_{k 1}^{2}, D_{k 2}^{1}$ and $D_{k 2}^{2}$ are parameters of the controller $\mathcal{K}$ given by

$$
\mathcal{K}:=\left[\begin{array}{c|cc}
A_{k}-s E_{k} & B_{k 1} & B_{k 2} \\
\hline C_{k}^{1} & D_{k 1}^{1} & D_{k 2}^{1} \\
C_{k}^{2} & D_{k 1}^{2} & D_{k 2}^{2}
\end{array}\right],
$$

which internally stabilizes the auxiliary system $\Sigma$ of the form

$$
\Sigma:=\left[\begin{array}{c|c|c}
A-s E & {\left[\begin{array}{c|c}
B_{2} & X_{i} \\
& I_{n_{i}}
\end{array}\right]} \\
\hline\left[\begin{array}{cc}
C_{2} \\
\hline I_{n_{o}} Y_{o}
\end{array}\right] & {\left[\begin{array}{cc}
0 & 0 \\
0 & 0
\end{array}\right]}
\end{array} .\right.
$$


Proof. We show that the two generalized Sylvester-type equations (8) and (9) are necessary for the existence of a solution to Conditions (C1) and (C'2). For the partition (6), by Lemma 5.3 (page 71) of Feng and Yagoubi (2017), there exist matrices $X_{i} \in \mathbb{R}^{\left(n+n_{o}\right) \times n_{i}}, Y_{i} \in \mathbb{R}^{\left(n+n_{o}\right) \times n_{i}}$ and $\Upsilon \in \mathbb{R}^{n_{k} \times n_{i}}$ such that

$$
\begin{aligned}
0 & =D_{32}\left(D_{k} \bar{C}_{22}-D_{k} \bar{C}_{21} Y_{i}-C_{k} \Upsilon\right)-\bar{C}_{31} Y_{i}+\bar{C}_{32}, \\
-X_{i} A_{i} & =\bar{A}_{11} Y_{i}+\bar{B}_{12}\left(D_{k} \bar{C}_{21} Y_{i}+C_{k} \Upsilon-D_{k} \bar{C}_{22}\right)-\bar{A}_{12}, \\
-X_{i} & =\bar{E} Y_{i} .
\end{aligned}
$$

Defining $\Pi_{i}=D_{k} \bar{C}_{21} Y_{i}+C_{k} \Upsilon-D_{k} \bar{C}_{22}$, the generalized Sylvester-type equation (8) holds. (9) can be proven by the same token for the partition (7).

Sufficiency is achieved in such a way: through the two generalized Sylvester-type equations (8) and (9), together with certain transformation matrices, we show that the structured controller (10) eliminates the unstabilizable and undetectable dynamics introduced by the exo-system $W_{i}$ and output weight $W_{o}$ in the closed-loop system. The multiobjective problem is equivalent to a standard problem for an auxiliary system constructed from the data of the weighted system.

To this end, we first reform the controller (10) as

$$
K:=\left[\begin{array}{cc|c}
A_{i}+D_{k 1}^{2} \Gamma_{i}-s I_{n_{i}} & \bar{C}_{k 2} & D_{k 1}^{2} \\
\bar{B}_{k} \Gamma_{i} & \bar{A}_{k}-s \bar{E}_{k} & \bar{B}_{k} \\
\hline \Omega_{i} & \bar{C}_{k 1} & D_{k 1}^{1}
\end{array}\right],
$$

where

$$
\begin{array}{ll}
\bar{E}_{k}=\left[\begin{array}{cc}
E_{k} & 0 \\
0 & E_{o}
\end{array}\right], \quad \bar{A}_{k}=\left[\begin{array}{cc}
A_{k} & B_{k 2} \\
-\Gamma_{o} C_{k} & A_{o}-\Gamma_{o} D_{k 2}
\end{array}\right], \quad \bar{B}_{k}=\left[\begin{array}{c}
B_{k 1}, \\
\Omega_{o}
\end{array}\right], \\
\bar{C}_{k 1}=\left[\begin{array}{ll}
C_{k}^{1} & D_{k 2}^{1}
\end{array}\right], \quad \bar{C}_{k 2}=\left[\begin{array}{ll}
C_{k}^{2} & D_{k 2}^{2}
\end{array}\right] .
\end{array}
$$

The closed-loop system $G_{C L}$ formed by $G$ and $K$ is thus given as follows

$$
G_{C L}:=\left[\begin{array}{c|c}
A_{C}-s E_{C} & B_{C} \\
\hline C_{C 1} & D_{C 1} \\
C_{C 3} & D_{C 3}
\end{array}\right]
$$

where

$$
\begin{aligned}
& A_{C}=\left[\begin{array}{cccc}
\bar{A}_{11}+\bar{B}_{12} D_{k 1}^{1} \bar{C}_{21} & \bar{A}_{12}+\bar{B}_{12} D_{k 1}^{1} \bar{C}_{22} & \bar{B}_{12} \Omega_{i} & \bar{B}_{12} \bar{C}_{k 1} \\
0 & A_{i} & 0 & 0 \\
D_{k 1}^{2} \bar{C}_{21} & D_{k 1}^{2} \bar{C}_{22} & A_{i}+D_{k 1}^{2} \Gamma_{i} & \bar{C}_{k 2} \\
\bar{B}_{k} \bar{C}_{21} & \bar{B}_{k} \bar{C}_{22} & \bar{B}_{k} \Gamma_{i} & \bar{A}_{k}
\end{array}\right], \\
& E_{C}=\left[\begin{array}{cccc}
\bar{E} & 0 & 0 & 0 \\
0 & I_{n_{i}} & 0 & 0 \\
0 & 0 & I_{n_{i}} & 0 \\
0 & 0 & 0 & \bar{E}_{k}
\end{array}\right], \quad B_{C}=\left[\begin{array}{c}
\bar{B}_{11}+\bar{B}_{12} D_{k 1}^{1} D_{21} \\
0 \\
D_{k 1}^{2} D_{21} \\
\bar{B}_{k} D_{21}
\end{array}\right], \\
& C_{C 1}=\left[\begin{array}{llll}
\bar{C}_{11}+D_{12} D_{k 1}^{1} \bar{C}_{21} & \bar{C}_{12}+D_{12} D_{k 1}^{1} \bar{C}_{22} & D_{12} \Omega_{i} & D_{12} \bar{C}_{k 1}
\end{array}\right], \\
& C_{C 3}=\left[\begin{array}{llll}
\bar{C}_{31}+D_{32} D_{k 1}^{1} \bar{C}_{21} & \bar{C}_{32}+D_{32} D_{k 1}^{1} \bar{C}_{22} & D_{32} \Omega_{i} & D_{32} \bar{C}_{k 1}
\end{array}\right], \\
& D_{C 1}=D_{11}+D_{12} D_{k 1}^{1} D_{21}, \quad D_{C 3}=D_{31}+D_{32} D_{k 1}^{1} D_{21} \text {. }
\end{aligned}
$$


With the solution to (8), we define the following transformation matrices

$$
M_{1}=\left[\begin{array}{cccc}
I_{n+n_{o}} & -X_{i} & 0 & 0 \\
0 & I_{n_{i}} & 0 & 0 \\
0 & I_{n_{i}} & I_{n_{i}} & 0 \\
0 & 0 & 0 & I_{n_{k}}
\end{array}\right], \quad N_{1}=\left[\begin{array}{cccc}
I_{n+n_{o}} & -Y_{i} & 0 & 0 \\
0 & I_{n_{i}} & 0 & 0 \\
0 & -I_{n_{i}} & I_{n_{i}} & 0 \\
0 & 0 & 0 & I_{n_{k}}
\end{array}\right]
$$

Hence, we have

$$
\begin{aligned}
& M_{1} A_{C} N_{1}=\left[\begin{array}{cccc}
\bar{A}_{11}+\bar{B}_{12} D_{k 1}^{1} \bar{C}_{21} & \phi_{1} & \bar{B}_{12} \Omega_{i} & \bar{B}_{12} \bar{C}_{k 1} \\
0 & A_{i} & 0 & 0 \\
D_{k 1}^{2} \bar{C}_{21} & \phi_{2} & A_{i}+D_{k 1}^{2} \Gamma_{i} & \bar{C}_{k 2} \\
\bar{B}_{k} \bar{C}_{21} & \phi_{3} & \bar{B}_{k} \Gamma_{i} & \bar{A}_{k}
\end{array}\right], \\
& M_{1} E_{C} N_{1}=E_{C}, \quad M_{1} B_{C}=\left[\begin{array}{c}
\bar{B}_{11}+\bar{B}_{12} D_{k 1}^{1} D_{21} \\
0 \\
D_{k 1}^{2} D_{21} \\
\bar{B}_{k} D_{21}
\end{array}\right] \text {, } \\
& C_{C 3} N_{1}=\left[\begin{array}{llll}
\bar{C}_{31}+D_{32} D_{k 1}^{1} \bar{C}_{21} & \phi_{4} & D_{32} \Omega_{i} & D_{32} \bar{C}_{k 1}
\end{array}\right], \\
& C_{C 1} N_{1}=\left[\begin{array}{llll}
\bar{C}_{11}+D_{12} D_{k 1}^{1} \bar{C}_{21} & \phi_{5} & D_{12} \Omega_{i} & D_{12} \bar{C}_{k 1}
\end{array}\right] \text {, }
\end{aligned}
$$

where

$$
\begin{aligned}
& \phi_{1}=-\bar{A}_{11} Y_{i}+\bar{A}_{12}-X_{i} A_{i}+\bar{B}_{12}\left(D_{k 1}^{1} \bar{C}_{22}-D_{k 1}^{1} \bar{C}_{21} Y_{i}-\Omega_{i}\right)=0, \\
& \phi_{2}=D_{k 1}^{2}\left(\bar{C}_{22}-\bar{C}_{21} Y_{i}-\Gamma_{i}\right)=0, \quad \phi_{3}=\bar{B}_{k}\left(\bar{C}_{22}-\bar{C}_{21} Y_{i}-\Gamma_{i}\right)=0, \\
& \phi_{4}=-\bar{C}_{31} Y_{i}+\bar{C}_{32}+D_{32}\left(D_{k 1}^{1} \bar{C}_{22}-D_{k 1}^{1} \bar{C}_{21} Y_{i}-\Omega_{i}\right)=0, \\
& \phi_{5}=-\bar{C}_{11} Y_{i}+\bar{C}_{12}-D_{12} \Pi_{i},
\end{aligned}
$$

in the light of (8). Therefore, the closed-loop system $G_{C L}$ is restricted system equivalent to $\bar{G}_{C L}$ of the form

$$
\bar{G}_{C L}:=\left[\begin{array}{c|c}
\bar{A}_{C}-s \bar{E}_{C} & \bar{B}_{C} \\
\hline C_{C 1} & D_{C 1} \\
\bar{C}_{C 3} & D_{C 3}
\end{array}\right],
$$

where

$$
\begin{aligned}
& \bar{A}_{C}=\left[\begin{array}{cccc}
\bar{A}_{11}+\bar{B}_{12} D_{k 1}^{1} \bar{C}_{21} & \bar{B}_{12} \Omega_{i} & \bar{B}_{12} \bar{C}_{k 1} & 0 \\
D_{k 1}^{2} \bar{C}_{21} & A_{i}+D_{k 1}^{2} \Gamma_{i} & \bar{C}_{k 2} & 0 \\
\bar{B}_{k} \bar{C}_{21} & \bar{B}_{k} \Gamma_{i} & \bar{A}_{k} & 0 \\
0 & 0 & 0 & A_{i}
\end{array}\right], \\
& \bar{E}_{C}=\left[\begin{array}{cccc}
\bar{E} & 0 & 0 & 0 \\
0 & I_{n_{i}} & 0 & 0 \\
0 & 0 & \bar{E}_{k} & 0 \\
0 & 0 & 0 & I_{n_{i}}
\end{array}\right], \quad \bar{B}_{C}=\left[\begin{array}{c}
\bar{B}_{11}+\bar{B}_{12} D_{k 1}^{1} D_{21} \\
D_{k 1}^{2} D_{21} \\
\bar{B}_{k} D_{21} \\
0
\end{array}\right] \text {, } \\
& \bar{C}_{C 1}=\left[\begin{array}{llll}
\bar{C}_{11}+D_{12} D_{k 1}^{1} \bar{C}_{21} & D_{12} \Omega_{i} & D_{12} \bar{C}_{k 1} & \phi_{5}
\end{array}\right] \text {, } \\
& \bar{C}_{C 3}=\left[\begin{array}{llll}
\bar{C}_{31}+D_{32} D_{k 1}^{1} \bar{C}_{21} & D_{32} \Omega_{i} & D_{32} \bar{C}_{k 1} & 0
\end{array}\right] \text {. }
\end{aligned}
$$


It is observed that this system can be rewritten by $\mathcal{F}_{l}(\bar{G}, \bar{K})$, where

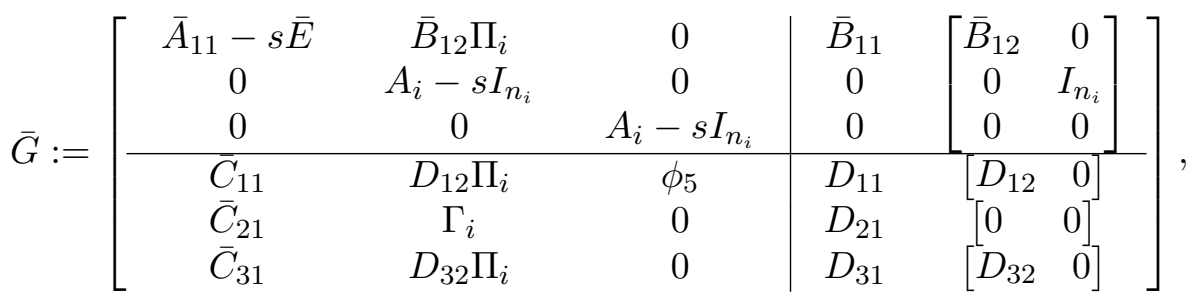

$$
\begin{aligned}
& \bar{K}:=\left[\begin{array}{c|c}
\bar{A}_{k}-s \bar{E}_{k} & \bar{B}_{k} \\
\hline \bar{C}_{k 1} & D_{k 1}^{1} \\
\bar{C}_{k 2} & D_{k 1}^{2}
\end{array}\right] \text {, }
\end{aligned}
$$

and the second output of $\bar{G}$ is the measurement. Then, using the two transformation matrices as follows

$$
M_{2}=\left[\begin{array}{ccc}
I_{n+n_{o}} & X_{i} & 0 \\
0 & I_{n_{i}} & 0 \\
0 & 0 & I_{n_{i}}
\end{array}\right], \quad N_{2}=\left[\begin{array}{ccc}
I_{n+n_{o}} & Y_{i} & 0 \\
0 & I_{n_{i}} & 0 \\
0 & 0 & I_{n_{i}}
\end{array}\right]
$$

the system $\bar{G}$ is restricted system equivalent to $\hat{G}$ of the form

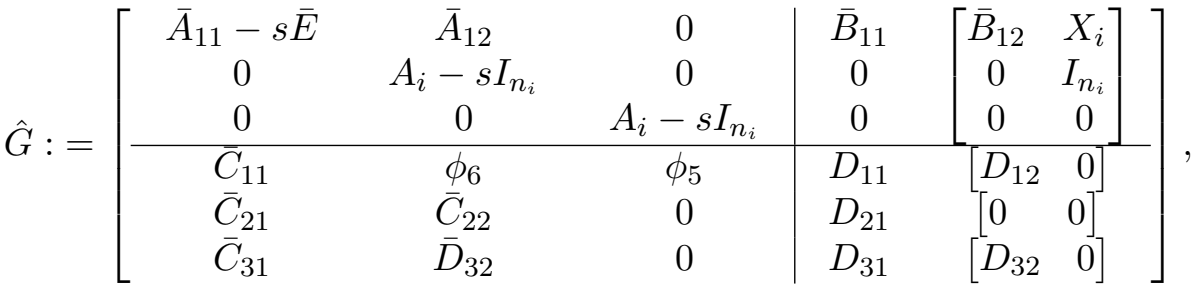

$$
\begin{aligned}
& =\left[\begin{array}{ccc|cc}
A_{o}-s E_{o} & \hat{A}_{12} & 0 & \hat{B}_{11} & \tilde{B}_{12} \\
0 & \hat{A}_{22}-s I_{n+n_{i}} & 0 & \hat{B}_{21} & \tilde{B}_{22} \\
0 & 0 & A_{i}-s I_{n_{i}} & 0 & 0 \\
\hline C_{o} & \tilde{C}_{12} & \phi_{5} & D_{11} & \tilde{D}_{12} \\
0 & \hat{C}_{22} & 0 & D_{21} & 0 \\
0 & \hat{C}_{32} & 0 & D_{31} & \tilde{D}_{32}
\end{array}\right] \text {, }
\end{aligned}
$$

where $\tilde{C}_{12}=\left[\begin{array}{ll}D_{o} C_{v} & \phi_{6}\end{array}\right], \phi_{6}=\bar{C}_{11} Y_{i}+D_{12} \Pi_{i}, \tilde{D}_{12}=\left[\begin{array}{ll}D_{12} & 0\end{array}\right], \tilde{D}_{32}=\left[\begin{array}{ll}D_{32} & 0\end{array}\right]$, and other data are given in (7) and (10). Then, the closed-loop system $\bar{G}_{C L}$ is further restricted system equivalent to $\hat{G}_{C L}=\mathcal{F}_{l}(\hat{G}, \bar{K})$ given by

$$
\hat{G}_{C L}:=\left[\begin{array}{c|c}
\hat{A}_{C}-s \hat{E}_{C} & \hat{B}_{C} \\
\hline \hat{C}_{C 1} & \hat{D}_{C 1} \\
\hat{C}_{C 3} & \hat{D}_{C 3}
\end{array}\right],
$$


where

$$
\begin{aligned}
& \hat{A}_{C}=\left[\begin{array}{ccccc}
A_{o} & \hat{A}_{12}+\tilde{B}_{12} D_{k 1} \hat{C}_{22} & \tilde{B}_{12} C_{k} & \tilde{B}_{12} D_{k 2} & 0 \\
0 & \hat{A}_{22}+\tilde{B}_{22} D_{k 1} \hat{C}_{22} & \tilde{B}_{22} C_{k} & \tilde{B}_{22} D_{k 2} & 0 \\
0 & B_{k 1} \hat{C}_{22} & A_{k} & B_{k 2} & 0 \\
0 & \Omega_{o} \hat{C}_{22} & -\Gamma_{o} C_{k} & A_{o}-\Gamma_{o} D_{k 2} & 0 \\
0 & 0 & 0 & 0 & A_{i}
\end{array}\right], \\
& \hat{E}_{C}=\left[\begin{array}{ccccc}
E_{o} & 0 & 0 & 0 & 0 \\
0 & I_{n+n_{o}} & 0 & 0 & 0 \\
0 & 0 & E_{k} & 0 & 0 \\
0 & 0 & 0 & E_{o} & 0 \\
0 & 0 & 0 & 0 & I_{n_{i}}
\end{array}\right], \quad \hat{B}_{C}=\left[\begin{array}{c}
\hat{B}_{11}+\tilde{B}_{12} D_{k 1} D_{21} \\
\hat{B}_{21}+\tilde{B}_{22} D_{k 1} D_{21} \\
B_{k 1} D_{21} \\
\Omega_{o} D_{21} \\
0
\end{array}\right] \text {, } \\
& \hat{C}_{C 1}=\left[\begin{array}{lllll}
C_{o} & \tilde{C}_{12}+\tilde{D}_{12} D_{k 1} \hat{C}_{22} & \tilde{D}_{12} C_{k} & \tilde{D}_{12} D_{k 2} & \phi_{5}
\end{array}\right] \text {, } \\
& \hat{C}_{C 3}=\left[\begin{array}{lllll}
0 & \hat{C}_{32}+\tilde{D}_{32} D_{k 1} \hat{C}_{22} & \tilde{D}_{32} C_{k} & \tilde{D}_{32} D_{k 2} & 0
\end{array}\right] \text {, } \\
& \hat{D}_{C 1}=D_{11}+\tilde{D}_{12} D_{k 1} D_{21}, \quad \hat{D}_{C 3}=D_{31}+\tilde{D}_{32} D_{k 1} D_{21} \text {. }
\end{aligned}
$$

Similarly, with the solution to (9), we define the following transformation matrices

$$
M_{3}=\left[\begin{array}{ccccc}
I_{n_{o}} & X_{o} & 0 & -I_{n_{o}} & 0 \\
0 & I_{n+n_{i}} & 0 & 0 & 0 \\
0 & 0 & I_{n_{k}} & 0 & 0 \\
0 & 0 & 0 & I_{n_{o}} & 0 \\
0 & 0 & 0 & 0 & I_{n_{i}}
\end{array}\right], \quad N_{3}=\left[\begin{array}{ccccc}
I_{n_{o}} & -Y_{o} & 0 & I_{n_{o}} & 0 \\
0 & I_{n+n_{i}} & 0 & 0 & 0 \\
0 & 0 & I_{n_{k}} & 0 & 0 \\
0 & 0 & 0 & I_{n_{o}} & 0 \\
0 & 0 & 0 & 0 & I_{n_{i}}
\end{array}\right] .
$$

Hence, there holds

$$
\begin{aligned}
& M_{3} \hat{A}_{C} N_{3}=\left[\begin{array}{ccccc}
A_{o} & \psi_{1} & \psi_{2} & \psi_{3} & 0 \\
0 & \hat{A}_{22}+\tilde{B}_{22} D_{k 1} \hat{C}_{22} & \tilde{B}_{22} C_{k} & \tilde{B}_{22} D_{k 2} & 0 \\
0 & B_{k 1} \hat{C}_{22} & A_{k} & B_{k 2} & 0 \\
0 & \Omega_{o} \hat{C}_{22} & -\Gamma_{o} C_{k} & A_{o}-\Gamma_{o} D_{k 2} & 0 \\
0 & 0 & 0 & 0 & A_{i}
\end{array}\right], \\
& M_{3} \hat{E}_{C} N_{3}=\hat{E}_{C}, \quad M_{3} \hat{B}_{C}=\left[\begin{array}{c}
\psi_{4} \\
\hat{B}_{21}+\tilde{B}_{22} D_{k 1} D_{21} \\
B_{k 1} D_{21} \\
\Omega_{o} D_{21} \\
0
\end{array}\right], \\
& \hat{C}_{C 1} N_{3}=\left[\begin{array}{lllll}
C_{o} & \tilde{C}_{12}+\tilde{D}_{12} D_{k 1} \hat{C}_{22}-C_{o} Y_{o} & \tilde{D}_{12} C_{k} & \tilde{D}_{12} D_{k 2}-C_{o} & \phi_{5}
\end{array}\right], \\
& \hat{C}_{C 3} N_{3}=\left[\begin{array}{llllll}
0 & \hat{C}_{32}+\tilde{D}_{32} D_{k 1} \hat{C}_{22} & \tilde{D}_{32} C_{k} & \tilde{D}_{32} D_{k 2} & 0
\end{array}\right] .
\end{aligned}
$$


where

$$
\begin{aligned}
& \psi_{1}=\hat{A}_{12}+\tilde{B}_{12} D_{k 1} \hat{C}_{22}-A_{o} Y_{o}+X_{o}\left(\hat{A}_{22}+\tilde{B}_{22} D_{k 1} \hat{C}_{22}\right)-\Omega_{o} \hat{C}_{22}=0, \\
& \psi_{2}=\left(\tilde{B}_{12}+X_{o} \tilde{B}_{22}+\Gamma_{o}\right) C_{k}=0 \\
& \psi_{3}=-A_{o}+\left(\tilde{B}_{12}+X_{o} \tilde{B}_{22}+\Gamma_{o}\right) C_{k}+A_{o}=0, \\
& \psi_{4}=\hat{B}_{11}+X_{o} \hat{B}_{21}+\left(\tilde{B}_{12} D_{k 1}+X_{o} \tilde{B}_{22} D_{k 1}-\Omega_{o}\right) D_{21}=0,
\end{aligned}
$$

in the light of (9). Hence, the closed-loop system can be written as

$$
\left\{\begin{aligned}
& {\left[\begin{array}{ccc}
I & 0 & 0 \\
0 & E_{k} & 0 \\
0 & 0 & E_{o}
\end{array}\right] \dot{x}_{c l}=} {\left[\begin{array}{ccc}
\hat{A}_{22}+\tilde{B}_{22} D_{k 1} \hat{C}_{22} & \tilde{B}_{22} C_{k} & \tilde{B}_{22} D_{k 2} \\
B_{k 1} \hat{C}_{22} & A_{k} & B_{k 2} \\
\Omega_{o} \hat{C}_{22} & -\Gamma_{o} C_{k} & A_{o}-\Gamma_{o} D_{k 2}
\end{array}\right] x_{c l} } \\
&+ {\left[\begin{array}{c}
\hat{B}_{21}+\tilde{B}_{22} D_{k 1} D_{21} \\
B_{k 1} D_{21} \\
\Omega_{o} D_{21}
\end{array}\right] w } \\
& \dot{x}_{i}=A_{i} x_{i}, \\
& z=\left[\begin{array}{lll}
\tilde{C}_{12}+\tilde{D}_{12} D_{k 1} \hat{C}_{22}-C_{o} Y_{o} & \tilde{D}_{12} C_{k} & \tilde{D}_{12} D_{k 2}+C_{o}
\end{array}\right] x_{c l}+\phi_{5} x_{i}+\hat{D}_{11} w \\
& e=\left[\begin{array}{lll}
\hat{C}_{32}+\tilde{D}_{32} D_{k 1} \hat{C}_{22} & \tilde{D}_{32} C_{k} & \tilde{D}_{32} D_{k 2}
\end{array}\right] x_{c l}+\hat{D}_{31} w .
\end{aligned}\right.
$$

It is observed that the exo-system $W_{i}$ neither affects the state $x_{c l}$ and the tracking error $e$, nor appears in the the transfer function $T_{z w}$. Hence, we can simply consider the following system instead

$$
\left\{\begin{aligned}
& {\left[\begin{array}{ccc}
I & 0 & 0 \\
0 & E_{k} & 0 \\
0 & 0 & E_{o}
\end{array}\right] \dot{x}_{c l}=} {\left[\begin{array}{ccc}
\hat{A}_{22}+\tilde{B}_{22} D_{k 1} \hat{C}_{22} & \tilde{B}_{22} C_{k} & \tilde{B}_{22} D_{k 2} \\
B_{k 1} \hat{C}_{22} & A_{k} & B_{k 2} \\
\Omega_{o} \hat{C}_{22} & -\Gamma_{o} C_{k} & A_{o}-\Gamma_{o} D_{k 2}
\end{array}\right] x_{c l} } \\
&+ {\left[\begin{array}{c}
\hat{B}_{21}+\tilde{B}_{22} D_{k 1} D_{21} \\
B_{k 1} D_{21} \\
\Omega_{o} D_{21}
\end{array}\right] w, } \\
& z=\left[\begin{array}{lll}
\tilde{C}_{12}+\tilde{D}_{12} D_{k 1} \hat{C}_{22}-C_{o} Y_{o} & \tilde{D}_{12} C_{k} & \tilde{D}_{12} D_{k 2}+C_{o}
\end{array}\right] x_{c l}+\hat{D}_{11} w, \\
& e=\left[\begin{array}{lll}
\hat{C}_{32}+\tilde{D}_{32} D_{k 1} \hat{C}_{22} & \tilde{D}_{32} C_{k} & \tilde{D}_{32} D_{k 2}
\end{array}\right] x_{c l}+\hat{D}_{31} w,
\end{aligned}\right.
$$

which can be further written by $\mathcal{F}_{l}(\bar{\Xi}, \mathcal{K})$, where $\mathcal{K}$ is given in $(11)$ and

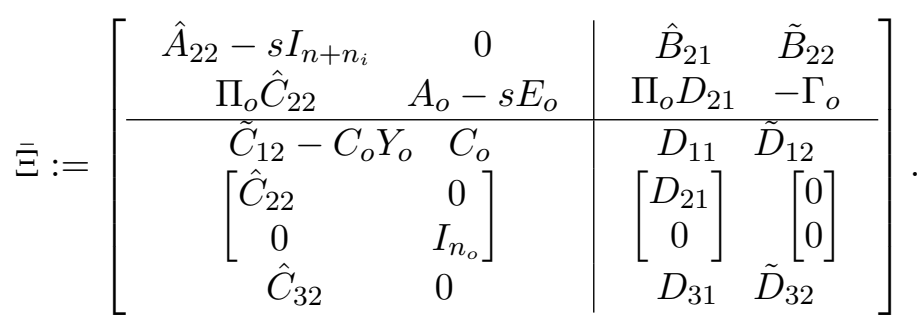

Using the two transformation matrices

$$
M_{4}=\left[\begin{array}{cc}
I_{n+n_{i}} & 0 \\
-X_{o} & I_{n_{o}}
\end{array}\right], \quad N_{4}=\left[\begin{array}{cc}
I_{n+n_{i}} & 0 \\
Y_{o} & I_{n_{o}}
\end{array}\right],
$$


one can show that $\bar{\Xi}$ is restricted system equivalent to $\Xi$ given by

$$
\Xi:=\left[\begin{array}{c|cc}
A-s E & B_{1} & {\left[\begin{array}{c|c}
B_{2} & X_{i} \\
I_{n_{i}}
\end{array}\right]} \\
\hline C_{1}\left[\begin{array}{cc}
I & Y_{i} \\
0 & 0
\end{array}\right]+\left[\begin{array}{ll}
0 & D_{12} \Pi_{i}
\end{array}\right] & D_{11} & {\left[\begin{array}{ll}
D_{12} & 0
\end{array}\right]} \\
{\left[\begin{array}{c}
C_{2} \\
I_{n_{o}} Y_{o}
\end{array}\right]} & {\left[\begin{array}{c}
D_{21} \\
0
\end{array}\right]} & {\left[\begin{array}{ll}
0 & 0 \\
0 & 0
\end{array}\right]} \\
D_{31} & {\left[\begin{array}{ll}
D_{32} & 0
\end{array}\right]}
\end{array}\right],
$$

where the second output is the measurement. It has been seen that $T_{z w}=\mathcal{F}_{l}(\bar{\Xi}, \mathcal{K})=$ $\mathcal{F}_{l}(\Xi, \mathcal{K})$ with the third output being removed. Note that $\mathcal{K}$ internally stabilizes the system $\Sigma$ in (12), which is indeed the system $\Xi_{22}$ induced from $\Xi$. Therefore, $T_{z w}$ is stable. Moreover, the tracking error $e$ is a linear combination of the state $x_{c l}$ and the external disturbance $w(t) \in \mathcal{L}_{2}$, hence, there holds $\lim _{t \rightarrow \infty} e(t)=0$.

In order to tackle the asymptotic tracking and nonproper output weighting filter, a structured measurement feedback controller is conducted and Fig. 2 exhibits explicitly the impact of the exo-system and output weight on this controller. An extra degree of freedom is also fully shown for the performance objective control, which will be discussed in the next section. Hence, Theorem 3.1 characterizes the set of all stabilizing controllers addressing asymptotic tracking and nonproper weights. The nonstandard problem is thus equivalent to a conventional control problem for the auxiliary system $\Xi$, which is constructed from the weighted system $G$ with the control input matrix and measurement output matrix being augmented by solutions to the two generalized Sylvester-type equations.

\section{Constrained $\mathcal{H}_{\infty}$ control}

In this section, relying on the paramererization of all stabilizing controllers given previously, the $\mathcal{H}_{\infty}$ performance control is tackled, and both full-order and reducedorder $\mathcal{H}_{\infty}$ controller synthese are studied in terms of LMI-based conditions. It has been shown that the structured controller (10) ensures asymptotic tracking and leads to a stable transfer function $T_{z w}$. Hence, the additional performance objective, i.e. Condition (C2) can be further approached with the benefit of the free controller $\mathcal{K}$ in (11). We assume that the two generalized Sylvester-type equations (8) and (9) admit solutions. As stated previously, Problem 2.1 is equivalent to an $\mathcal{H}_{\infty}$ control problem with respect to the axillary system $\Xi$ specified as follows

$$
\begin{aligned}
& \Xi:=\left[\begin{array}{c|cc}
A-s E & B_{1} & {\left[\begin{array}{c|c}
B_{2} & X_{i} \\
I_{n_{i}}
\end{array}\right]} \\
\hline C_{1}\left[\begin{array}{cc}
I & Y_{i} \\
0 & 0
\end{array}\right]+\left[\begin{array}{ll}
0 & D_{12} \Pi_{i}
\end{array}\right] & D_{11} & {\left[\begin{array}{ll}
D_{12} & 0
\end{array}\right]} \\
{\left[\begin{array}{cc}
\frac{C_{2}}{I_{n_{o}}} & Y_{o}
\end{array}\right]} & {\left[\begin{array}{c}
D_{21} \\
0
\end{array}\right]} & {\left[\begin{array}{ll}
0 & 0 \\
0 & 0
\end{array}\right]}
\end{array}\right] \\
& =\left[\begin{array}{c|cc}
A-s E & B_{1} & \mathcal{B}_{2}\left(X_{i}\right) \\
\hline \mathcal{C}_{1}\left(Y_{i}, \Pi_{i}\right) & D_{11} & \mathcal{D}_{12} \\
\mathcal{C}_{2}\left(Y_{o}\right) & \mathcal{D}_{21} & 0
\end{array}\right],
\end{aligned}
$$


with the third output being removed, since the tracking issue is guaranteed through the structured controller. Note that this system has invariant zeros at infinity, which is why the Riccati-based solution Wang, Yung, and Chang (2006) fails. Hence, we adopt the LMI-based approach to solve the underlying descriptor $\mathcal{H}_{\infty}$ control problem. Moreover, it is observed that the auxiliary system depends on the generalized Sylvestertype equations solutions, which are generally not unique. This fact indicates that the underlying controller design may result in a nonlinear matrix inequality problem, and proceeding with certain specific solution by directly applying existing methods may lead to a conservative synthesis. In this section, we fully explore the structure of the auxiliary system and present a design route independent of the solutions to generalized Sylvester-type equations.

Before presenting the controller synthesis, we assume without loss of generality that the matrix $E$ is of the form $E=\operatorname{diag}\left(I_{n+n_{i}+r_{o}}, 0_{\left(n_{o}-r_{o}\right) \times\left(n_{o}-r_{o}\right)}\right)$. Note that if $E$ is not of this form, then a pair of transformation matrices can easily be obtained due to a singular value decomposition (SVD) to make $E$ satisfy this condition.

\subsection{Full-Order $\mathcal{H}_{\infty}$ Controller}

Theorem 4.1. Recall the partitions $G^{W_{i}}$ and $G^{W_{o}}$ in (6) and (7). Given $\gamma>0$, there exists a measurement output controller (11) with $\operatorname{rank}\left(E_{k}\right)=n+n_{i}+r_{o}$ such that the $\mathcal{H}_{\infty}$ norm of the closed-loop system formed by $\Xi$ in (13) and $\mathcal{K}$ in (11) is bounded by $\gamma$, if and only if there exist matrices $X_{i} \in \mathbb{R}^{\left(n+n_{o}\right) \times n_{i}}, Y_{i} \in \mathbb{R}^{\left(n+n_{o}\right) \times n_{i}}, \Pi_{i} \in \mathbb{R}^{m \times n_{i}}$, $X_{o} \in \mathbb{R}^{n_{o} \times\left(n+n_{i}\right)}, Y_{o} \in \mathbb{R}^{n_{o} \times\left(n+n_{i}\right)}, \Pi_{o} \in \mathbb{R}^{n_{o} \times p}, \mathcal{X} \in \mathbb{R}^{\left(n+n_{i}+n_{o}\right) \times\left(n+n_{i}+n_{o}\right)}, \mathcal{Y} \in$ $\mathbb{R}^{\left(n+n_{i}+n_{o}\right) \times\left(n+n_{i}+n_{o}\right)}, \mathcal{W} \in \mathbb{R}^{\left(n+n_{i}+n_{o}\right) \times m}$, and $\mathcal{Z} \in \mathbb{R}^{m \times\left(n+n_{i}+n_{o}\right)}$ satisfying (8), (9), and

$$
\begin{aligned}
& {\left[\begin{array}{cc}
E^{T} \mathcal{X} & \bullet \\
E & E \mathcal{Y}^{T}
\end{array}\right]=\left[\begin{array}{cc}
\mathcal{X}^{T} E & \bullet \\
E & \mathcal{Y} E^{T}
\end{array}\right] \geq 0,} \\
& E^{T} \mathcal{W}=0, \quad E \mathcal{Z}^{T}=0 \\
& L_{c}(\bar{Y}, \bar{Z})<0,
\end{aligned}
$$

$$
L_{o}(\bar{X}, \bar{W})<0,
$$

where

$$
\begin{aligned}
& L_{c}(\bar{Y}, \bar{Z})=\left[\begin{array}{cc}
N_{c} & 0 \\
0 & I
\end{array}\right]^{T}\left[\begin{array}{ccc}
\boldsymbol{H e}\left\{\bar{A}_{11} \bar{Y}^{T}\right\} & \bullet & \bullet \\
\bar{C}_{11} \bar{Y}^{T} & -\gamma I & \bullet \\
\bar{Z} \bar{A}_{11}^{T}+\bar{B}_{11}^{T} & D_{11}^{T}+\bar{Z} \bar{C}_{11}^{T} & -\gamma I
\end{array}\right]\left[\begin{array}{cc}
N_{c} & 0 \\
0 & I
\end{array}\right] \\
& L_{o}(\bar{X}, \bar{W})=\left[\begin{array}{cc}
N_{o} & 0 \\
0 & I
\end{array}\right]^{T}\left[\begin{array}{ccc}
\boldsymbol{H e}\left\{\hat{A}_{22}^{T} \bar{X}\right\} & \bullet & \bullet \\
\bar{W}^{T} \hat{A}_{22}+\hat{B}_{21}^{T} \bar{X} & \boldsymbol{H e}\left\{\hat{B}_{21}^{T} \bar{W}\right\}-\gamma I & \bullet \\
\tilde{C}_{12}-C_{o} Y_{o} & D_{11} & -\gamma I
\end{array}\right]\left[\begin{array}{cc}
N_{o} & 0 \\
0 & I
\end{array}\right] \text {, } \\
& N_{c}=\left[\begin{array}{c}
\bar{B}_{12} \\
D_{12}
\end{array}\right]^{\perp}, \quad N_{o}=\left[\begin{array}{ll}
\hat{B}_{22} & D_{21}
\end{array}\right]^{\perp}, \quad \bar{X}=\left[\begin{array}{ll}
0 & I_{n+n_{i}}
\end{array}\right] \mathcal{X}\left[\begin{array}{c}
0 \\
I_{n+n_{i}}
\end{array}\right] \text {, } \\
& \bar{Y}=\left[\begin{array}{ll}
I_{n+n_{o}} & 0
\end{array}\right] \mathcal{Y}\left[\begin{array}{c}
I_{n+n_{o}} \\
0
\end{array}\right], \quad \bar{W}=\left[\begin{array}{ll}
0 & I_{n+n_{i}}
\end{array}\right] \mathcal{W}, \quad \bar{Z}=\mathcal{Z}\left[\begin{array}{c}
I_{n+n_{o}} \\
0
\end{array}\right] \text {, } \\
& \tilde{C}_{12}=\left[\begin{array}{ll}
D_{o} C_{v} & \bar{C}_{11} Y_{i}+D_{12} \Pi_{i}
\end{array}\right] \text {. }
\end{aligned}
$$


Proof. By Theorem 1 in Masubuchi (2007), the $\mathcal{H}_{\infty}$ controller exists if and only if there exist matrices $\mathcal{X}, \mathcal{Y}, \mathcal{W}$ and $\mathcal{Z}$ with appropriate dimensions satisfying (14), (15), and

$$
\begin{aligned}
& {\left[\begin{array}{cc}
\mathcal{N}_{c} & 0 \\
0 & I
\end{array}\right]^{T} \Theta_{c}\left[\begin{array}{cc}
\mathcal{N}_{c} & 0 \\
0 & I
\end{array}\right]<0,} \\
& {\left[\begin{array}{cc}
\mathcal{N}_{o} & 0 \\
0 & I
\end{array}\right]^{T} \Theta_{o}\left[\begin{array}{cc}
\mathcal{N}_{o} & 0 \\
0 & I
\end{array}\right]<0,}
\end{aligned}
$$

where

$$
\begin{gathered}
\mathcal{N}_{c}=\left[\begin{array}{c}
\mathcal{B}_{2}\left(X_{i}\right) \\
\mathcal{D}_{12}
\end{array}\right]^{\perp}, \quad \Theta_{c}=\left[\begin{array}{ccc}
\operatorname{He}\left\{A \mathcal{Y}^{T}\right\} & \bullet & \bullet \\
\mathcal{C}_{1}\left(Y_{i}, \Pi_{i}\right) \mathcal{Y}^{T} & -\gamma I & \bullet \\
B_{1}^{T}+\mathcal{Z} A^{T} & D_{11}^{T}+\mathcal{Z} \mathcal{C}_{1}\left(Y_{i}, \Pi_{i}\right) & -\gamma I
\end{array}\right], \\
\mathcal{N}_{o}=\left[\begin{array}{ll}
\mathcal{C}_{2}\left(Y_{o}\right) & \mathcal{D}_{21}
\end{array}\right]^{\perp}, \quad \Theta_{o}=\left[\begin{array}{ccc}
\operatorname{He}\left\{A^{T} \mathcal{X}\right\} & \bullet & \bullet \\
B_{1}^{T} \mathcal{X}+\mathcal{W}^{T} A & \operatorname{He}\left\{B_{1}^{T} \mathcal{W}\right\}-\gamma I & \bullet \\
\mathcal{C}_{1}\left(Y_{i}, \Pi_{i}\right) & D_{11} & -\gamma I
\end{array}\right] .
\end{gathered}
$$

Note that $\Theta_{c}$ contains a multiplication between the decision variable $\mathcal{Y}$ and the auxiliary system data $\mathcal{C}_{1}\left(Y_{i}, \Pi_{i}\right)$ depending on both $Y_{i}$ and $\Pi_{i}$. Hence, this condition is obviously not an LMI. Same observations hold for $\mathcal{N}_{c}$ and $\mathcal{N}_{o}$. In order to obtain linear conditions, let us set

$$
M_{i}=\left[\begin{array}{cc}
I_{n+n_{o}} & X_{i} \\
0 & I_{n_{i}}
\end{array}\right], \quad N_{i}=\left[\begin{array}{cc}
I_{n+n_{o}} & Y_{i} \\
0 & I_{n_{i}}
\end{array}\right]
$$

Note that

$$
\left[\begin{array}{c}
\mathcal{B}_{2}\left(X_{i}\right) \\
\mathcal{D}_{12}
\end{array}\right]=\left[\begin{array}{cc}
M_{i} & 0 \\
0 & I_{p_{o}}
\end{array}\right]\left[\begin{array}{cc}
\bar{B}_{12} & 0 \\
0 & I_{n_{i}} \\
D_{12} & 0
\end{array}\right]
$$

which implies

$$
\mathcal{N}_{c}=\left[\begin{array}{cc}
M_{i}^{-T} & 0 \\
0 & I_{p_{o}}
\end{array}\right]\left[\begin{array}{cc}
\bar{B}_{12} & 0 \\
0 & I_{n_{i}} \\
D_{12} & 0
\end{array}\right]^{\perp}=\left[\begin{array}{cc}
M_{i}^{-T} & 0 \\
0 & I_{p_{o}}
\end{array}\right]\left[\begin{array}{c}
N_{c 1} \\
0 \\
N_{c 2}
\end{array}\right]
$$

where $\left[\begin{array}{l}N_{c 1} \\ N_{c 2}\end{array}\right]=\left[\begin{array}{c}\bar{B}_{12} \\ D_{12}\end{array}\right]^{\perp}$. Then, the condition (18) is equivalent to

$$
\left[\begin{array}{cc}
N_{c 1} & 0 \\
0 & 0 \\
N_{c 2} & 0 \\
0 & I
\end{array}\right]^{T}\left[\begin{array}{cc}
M_{i}^{-1} & 0 \\
0 & I_{p_{o}}
\end{array}\right] \Theta_{c}\left[\begin{array}{cc}
M_{i}^{-T} & 0 \\
0 & I_{p_{o}}
\end{array}\right]\left[\begin{array}{cc}
N_{c 1} & 0 \\
0 & 0 \\
N_{c 2} & 0 \\
0 & I
\end{array}\right]<0 .
$$


Set $Y=M_{i}^{-1} \mathcal{Y} N_{i}^{T}$ and $Z=\mathcal{Z} N_{i}^{T}$. Denote the partitions as follows

$$
Y=\left[\begin{array}{cc}
\bar{Y} & Y_{12} \\
Y_{21} & Y_{22}
\end{array}\right], \quad Z=\left[\begin{array}{ll}
\bar{Z} & Z_{2}
\end{array}\right]
$$

Then, the condition (18) can be further rewritten by

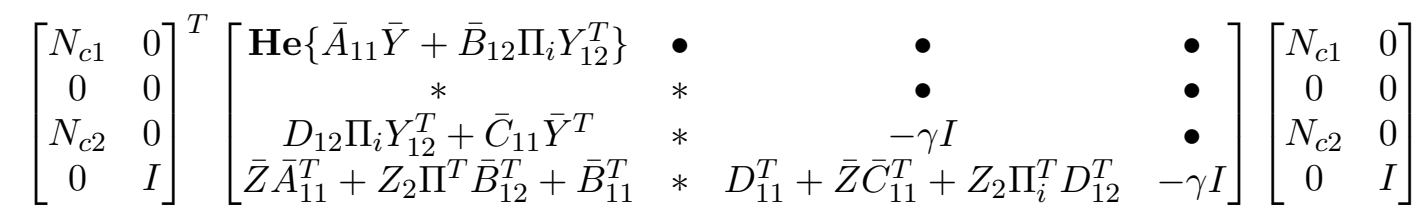

$$
\begin{aligned}
& =\left[\begin{array}{cc}
N_{c 1} & 0 \\
N_{c 2} & 0 \\
0 & I
\end{array}\right]^{T}\left[\begin{array}{ccc}
\mathbf{H e}\left\{\bar{A}_{11} \bar{Y}+\bar{B}_{12} \Pi_{i} Y_{12}^{T}\right\} & \bullet & \bullet \\
D_{12} \Pi_{i} Y_{12}^{T}+\bar{C}_{11} \bar{Y}^{T} & -\gamma I & \bullet \\
\bar{Z} \bar{A}_{11}^{T}+Z_{2} \Pi^{T} \bar{B}_{12}^{T}-\bar{B}_{11}^{T} & D_{11}^{T}+\bar{Z} \bar{C}_{11}^{T}+Z_{2} \Pi_{i}^{T} D_{12}^{T} & -\gamma I
\end{array}\right]\left[\begin{array}{cc}
N_{c 1} & 0 \\
N_{c 2} & 0 \\
0 & I
\end{array}\right] \\
& =L_{c}(\bar{Y}, \bar{Z})+\left[\begin{array}{cc}
N_{c 1} & 0 \\
N_{c 2} & 0 \\
0 & I
\end{array}\right]^{T}\left[\begin{array}{ccc}
\mathbf{H e}\left\{\bar{B}_{12} \Pi_{i} Y_{12}^{T}\right\} & \bullet & \bullet \\
D_{12} \Pi_{i} Y_{12}^{T} & 0 & \bullet \\
Z_{2} \Pi^{T} \bar{B}_{12}^{T} & Z_{2} \Pi_{i}^{T} D_{12}^{T} & 0
\end{array}\right]\left[\begin{array}{cc}
N_{c 1} & 0 \\
N_{c 2} & 0 \\
0 & I
\end{array}\right]<0,
\end{aligned}
$$

where ' $*$ ' stands for the elements irrelevant to the discussion. Since $N_{c 1}^{T} \bar{B}_{12}+N_{c 2}^{T} D_{12}=$ 0 , the second term of the last inequality is zero. Hence, the condition (16) holds. Similarly, let us set

$$
M_{o}=\left[\begin{array}{cc}
I_{n_{o}} & -X_{o} \\
0 & I_{n+n_{i}}
\end{array}\right], \quad N_{o}=\left[\begin{array}{cc}
I_{n_{o}} & Y_{o} \\
0 & I_{n+n_{i}}
\end{array}\right] .
$$

Note that

$$
\left[\mathcal{C}_{2}\left(Y_{o}\right) \quad \mathcal{D}_{21}\right]=\left[\begin{array}{ccc}
0 & \hat{C}_{22} & D_{21} \\
I_{n_{o}} & 0 & 0
\end{array}\right]\left[\begin{array}{cc}
N_{o} & 0 \\
0 & I_{p}
\end{array}\right]
$$

which implies

$$
\mathcal{N}_{o}=\left[\begin{array}{cc}
N_{o}^{-1} & 0 \\
0 & I_{p}
\end{array}\right]\left[\begin{array}{cc}
0 & I_{n_{o}} \\
\hat{C}_{22}^{T} & 0 \\
D_{21}^{T} & 0
\end{array}\right]^{\perp}=\left[\begin{array}{cc}
N_{o}^{-1} & 0 \\
0 & I_{p}
\end{array}\right]\left[\begin{array}{c}
0 \\
N_{o 1} \\
N_{o 2}
\end{array}\right]
$$

where $\left[\begin{array}{l}N_{o 1} \\ N_{o 2}\end{array}\right]=\left[\begin{array}{l}\hat{C}_{22}^{T} \\ D_{21}^{T}\end{array}\right]^{\perp}$. Then, the condition (19) is equivalent to

$$
\left[\begin{array}{cc}
0 & 0 \\
N_{o 1} & 0 \\
N_{o 2} & 0 \\
0 & I
\end{array}\right]^{T}\left[\begin{array}{cc}
N_{o}^{-T} & 0 \\
0 & I_{p}
\end{array}\right] \Theta_{o}\left[\begin{array}{cc}
N_{o}^{-1} & 0 \\
0 & I_{p}
\end{array}\right]\left[\begin{array}{cc}
0 & 0 \\
N_{o 1} & 0 \\
N_{o 2} & 0 \\
0 & I
\end{array}\right]<0 .
$$

Set $X=M_{o}^{T} \mathcal{X} N_{o}^{-1}$ and $W=M_{o}^{T} \mathcal{W}$. Denote the partitions as follows

$$
X=\left[\begin{array}{cc}
X_{11} & X_{12} \\
X_{21} & \bar{X}
\end{array}\right], \quad W=\left[\begin{array}{l}
W_{1} \\
\bar{W}
\end{array}\right] .
$$


Then, the condition (19) can be further rewritten by

$$
\begin{aligned}
& {\left[\begin{array}{cc}
0 & 0 \\
N_{o 1} & 0 \\
N_{o 2} & 0 \\
0 & I
\end{array}\right]^{T}\left[\begin{array}{cccc}
* & \mathbf{H e}\left\{\hat{A}_{22} \bar{X}+X_{12}^{T} \Pi_{o} \hat{C}_{22}\right\} & \bullet & \bullet \\
* & \hat{B}_{21}^{T} \bar{X}+D_{21}^{T} \Pi_{o}^{T} X_{12}+W_{1}^{T} \Pi_{o} \hat{C}_{22} & \mathbf{H e}\left\{W_{1}^{T} \Pi_{o} D_{21}+\hat{B}_{21} \bar{W}\right\}-\gamma I & \bullet \\
* & \tilde{C}_{12}-C_{o} Y_{o} & D_{11} & -\gamma I
\end{array}\right]\left[\begin{array}{cc}
0 & 0 \\
N_{o 1} & 0 \\
N_{o 2} & 0 \\
0 & I
\end{array}\right] } \\
& {\left[\begin{array}{cc}
N_{o 1} & 0 \\
N_{o 2} & 0 \\
0 & I
\end{array}\right]^{T}\left[\begin{array}{ccc}
\mathbf{H e}\left\{\hat{A}_{22} \bar{X}+X_{12}^{T} \Pi_{o} \hat{C}_{22}\right\} & \bullet & \bullet \\
\hat{B}_{21}^{T} \bar{X}+D_{21}^{T} \Pi_{o}^{T} X_{12}+W_{1}^{T} \Pi_{o} \hat{C}_{22} & \mathbf{H e}\left\{W_{1}^{T} \Pi_{o} D_{21}+\hat{B}_{21} \bar{W}\right\}-\gamma I & \bullet \\
\tilde{C}_{12}-C_{o} Y_{o} & D_{11} & -\gamma I
\end{array}\right]\left[\begin{array}{cc}
N_{o 1} & 0 \\
N_{o 2} & 0 \\
0 & I
\end{array}\right] } \\
= & L_{o}(\bar{X}, \bar{W})+\left[\begin{array}{cc}
N_{o 1} & 0 \\
N_{o 2} & 0 \\
0 & I
\end{array}\right]^{T}\left[\begin{array}{ccc}
\mathbf{H e}\left\{X_{12}^{T} \Pi_{o} \hat{C}_{22}\right\} & \bullet & \bullet \\
D_{21}^{T} \Pi_{o}^{T} X_{12}+W_{1}^{T} \Pi_{o} \hat{C}_{22} & \mathbf{H e}\left\{W_{1}^{T} \Pi_{o} D_{21}\right\} & \bullet \\
0 & 0 & 0
\end{array}\right]\left[\begin{array}{cc}
N_{o 1} & 0 \\
N_{o 2} & 0 \\
0 & I
\end{array}\right]<0 .
\end{aligned}
$$

Since $N_{o 1}^{T} \hat{C}_{22}+N_{o 2}^{T} D_{21}^{T}=0$, the second term is zero. Hence, the condition (17) holds.

The LMI (14) implies that solutions $\mathcal{X}$ and $\mathcal{Y}$ are in the form of

$$
\mathcal{X}=\left[\begin{array}{cc}
\mathcal{X}_{p 11} & 0 \\
\mathcal{X}_{p 21} & \mathcal{X}_{p 22}
\end{array}\right], \quad \mathcal{Y}=\left[\begin{array}{cc}
\mathcal{Y}_{p 11} & \mathcal{Y}_{p 12} \\
0 & \mathcal{Y}_{p 22}
\end{array}\right]
$$

Furthermore, we define the following two matrices

$$
\mathcal{X}_{11}=\left[\begin{array}{cc}
\mathcal{X}_{p 11} & \mathcal{X}_{p c 11} \\
\mathcal{X}_{c p 11} & \mathcal{X}_{c 11}
\end{array}\right], \quad \mathcal{X}_{11}^{-1}=\mathcal{Y}_{11}=\left[\begin{array}{cc}
\mathcal{Y}_{p 11} & \mathcal{Y}_{p c 11} \\
\mathcal{Y}_{c p 11} & \mathcal{Y}_{c 11}
\end{array}\right]
$$

By the standard way for state-space $\mathcal{H}_{\infty}$ control Gahinet and Apkarian (1994), we have $\mathcal{X}_{p c 11} \mathcal{X}_{c 11}^{-1} \mathcal{X}_{c p 11}=\mathcal{X}_{p 11}-\mathcal{Y}_{p 11}^{-1}$. Then, we can obtain the closed-loop Lyapunov matrix as

$$
\mathcal{X}_{C L}=\left[\begin{array}{cc|cc}
\mathcal{X}_{p 11} & 0 & \mathcal{X}_{p c 11} & 0 \\
\mathcal{X}_{p 21} & \mathcal{X}_{p 22} & 0 & I_{n_{o}-r_{o}} \\
\hline \mathcal{X}_{c p 11} & 0 & \mathcal{X}_{c 11} & 0 \\
\mathcal{X}_{c p 21} & \mathcal{X}_{c p 22} & \mathcal{X}_{c 21} & -\mathcal{Y}_{p 22}^{T}
\end{array}\right]
$$

where $\mathcal{X}_{c p 21}=-\left(\mathcal{Y}_{p 22}^{T} \mathcal{X}_{p 21}+\mathcal{Y}_{p 12} \Delta^{-T}\right), \quad \mathcal{X}_{c p 22}=I-\mathcal{Y}_{p 22}^{T} \mathcal{X}_{p 22}, \quad \mathcal{X}_{c 21}=$ $\mathcal{Y}_{p 12}^{T} \Delta^{-T} \mathcal{Y}_{c p 11}^{T} \mathcal{Y}_{c 11}^{-T}, \Delta=\mathcal{Y}_{p 11}-\mathcal{Y}_{p c 11} \mathcal{Y}_{c 11}^{-1} \mathcal{Y}_{c p 11}$

Once the closed-loop Lyapunov matrix is done, we form the following matrices

$$
\begin{aligned}
& A_{0}=\left[\begin{array}{cc}
A & 0 \\
0 & 0
\end{array}\right], \quad B_{01}=\left[\begin{array}{c}
B_{1} \\
0
\end{array}\right], \quad B_{02}=\left[\begin{array}{cc}
0 & \mathcal{B}_{2}\left(X_{i}\right) \\
I & 0
\end{array}\right], \quad C_{01}=\left[\begin{array}{ll}
\mathcal{C}_{1}\left(Y_{i}, \Pi_{i}\right) & 0
\end{array}\right], \\
& C_{02}=\left[\begin{array}{cc}
0 & I \\
\mathcal{C}_{2}\left(Y_{o}\right) & 0
\end{array}\right], \quad D_{012}=\left[\begin{array}{ll}
0 & \mathcal{D}_{12}
\end{array}\right], \quad D_{021}=\left[\begin{array}{c}
0 \\
\mathcal{D}_{21}
\end{array}\right] .
\end{aligned}
$$

Then, the resulting $\mathcal{H}_{\infty}$ controller $\mathcal{K}$ can be obtained through solving the following 
LMI

$$
\left[\begin{array}{ccc}
A_{0}^{T} \mathcal{X}_{C L} & \bullet & \bullet \\
B_{0}^{T} \mathcal{X}_{C L} & -\gamma I & \bullet \\
C_{01} & D_{11} & -\gamma I
\end{array}\right]+\mathbf{H e}\left\{\mathcal{P}^{T}\left[\begin{array}{c|cc}
A_{k} & B_{k 1} & B_{k 2} \\
\hline C_{k}^{1} & D_{k 1}^{1} & D_{k 2}^{1} \\
C_{k}^{2} & D_{k 1}^{2} & D_{k 2}^{2}
\end{array}\right] \mathcal{Q}\right\}<0
$$

where $\mathcal{P}=\left[\begin{array}{lll}B_{02}^{T} \mathcal{X}_{C L} & 0 & D_{012}^{T}\end{array}\right]$ and $\mathcal{Q}=\left[\begin{array}{lll}C_{02} & D_{021} & 0\end{array}\right]$. Moreover, $E_{k}$ is set by $E_{k}=E=\operatorname{diag}\left(I_{n+n_{i}+r_{o}}, 0_{\left(n_{o}-r_{o}\right) \times\left(n_{o}-r_{o}\right)}\right)$.

Now, we are in a position to give a complete procedure to form a full-order controller solving Problem 2.1.

Algorithm 4.2 (Full-order Solution).

(1) Simultaneously solve the generalized Sylvester-type equations (8) and (9), and LMI conditions (14)-(17). Get solutions $\left(X_{i}, Y_{i}, \Pi_{i}, X_{o}, Y_{o}, \Pi_{o}\right)$ and $(\mathcal{X}, \mathcal{Y}, \mathcal{W}, \mathcal{Z})$

(2) Based on $\mathcal{X}$ and $\mathcal{Y}$, form the Lyapunov matrix $\mathcal{X}_{C L}(20)$, and obtain the $\mathcal{H}_{\infty}$ controller $\mathcal{K}(11)$ by solving (21).

(3) Construct the controller $K$ by (10) with $\left(X_{i}, Y_{i}, \Pi_{i}, X_{o}, Y_{o}, \Pi_{o}\right)$ and $\mathcal{K}$.

\subsection{Reduced-Order $\mathcal{H}_{\infty}$ Controller}

It is worthy of noting that the auxiliary system $\Xi$ given in (13) contains $n+n_{i}+r_{o}$ finite dynamic modes and $n_{o}-r_{o}$ infinite modes. In the parts to follow, for the sake of simplicity, let us abuse a bit the use of notation by calling the sum of finite and infinite modes as the order of system. Since the $\mathcal{H}_{\infty}$ controller obtained previously has the same order of the the auxiliary system $\Xi$, the overall controller $K$ given in (10) has the order $n+2 n_{i}+2 n_{o}$, which exceeds the order of the original generalized system $G$, and is obviously not desirable, especially for the case where higher order exo-systems or output weighting filters are involved. In order to conduct a solution that is easy to implement, we explore the issue of reduced-order controller synthesis in this subsection.

Consider the two systems, denoted respectively as $\Xi_{12}$ and $\Xi_{21}$, induced from the auxiliary system $\Xi$ as below

$$
\Xi_{12}=\left[\begin{array}{c|c}
A-s E & \mathcal{B}_{2}\left(X_{i}\right) \\
\hline \mathcal{C}_{1}\left(Y_{i}, \Pi_{i}\right) & {\left[\begin{array}{ll}
D_{12} & 0
\end{array}\right]}
\end{array}\right], \quad \Xi_{21}=\left[\begin{array}{c|c}
A-s E & B_{1} \\
\hline \mathcal{C}_{2}\left(Y_{o}\right) & {\left[\begin{array}{c}
D_{21} \\
0
\end{array}\right]}
\end{array}\right] .
$$

It is observed that both of them contain zeros at infinity, since the D-matrix of $\Xi_{12}$ is not full row rank, and the D-matrix of $\Xi_{21}$ is not full column rank. According to Xin, Hara, and Kaneda (2008), there exists a reduced-order $\mathcal{H}_{\infty}$ controller, with which the overall controller in (10) has an order less than $n+n_{i}+n_{o}$. The following theorem states a way to reduced-order controller synthesis.

Theorem 4.3. Given $\gamma>0$, there exists a measurement output feedback controller with an order less than $n$, such that the $\mathcal{H}_{\infty}$ norm of the closed-loop system formed by $\Xi$ and the controller is bounded by $\gamma$, if there exist matrices $X_{i} \in \mathbb{R}^{\left(n+n_{o}\right) \times n_{i}}$, $Y_{i} \in \mathbb{R}^{\left(n+n_{o}\right) \times n_{i}}, \Pi_{i} \in \mathbb{R}^{m \times n_{i}}, X_{o} \in \mathbb{R}^{n_{o} \times\left(n+n_{i}\right)}, Y_{o} \in \mathbb{R}^{n_{o} \times\left(n+n_{i}\right)}, \Pi_{o} \in \mathbb{R}^{n_{o} \times p}$, $\mathcal{X} \in \mathbb{R}^{\left(n+n_{i}+n_{o}\right) \times\left(n+n_{i}+n_{o}\right)}, \mathcal{Y} \in \mathbb{R}^{\left(n+n_{i}+n_{o}\right) \times\left(n+n_{i}+n_{o}\right)}, \mathcal{W} \in \mathbb{R}^{\left(n+n_{i}+n_{o}\right) \times m}, \mathcal{Z} \in$ 
$\mathbb{R}^{m \times\left(n+n_{i}+n_{o}\right)}$, and two positive scalars $\epsilon_{1}, \epsilon_{2}$ satisfying (8), (9), and

$$
\begin{aligned}
& {\left[\begin{array}{cc}
E^{T} \mathcal{X} & \bullet \\
E & E \mathcal{Y}^{T}
\end{array}\right]=\left[\begin{array}{cc}
\mathcal{X}^{T} E & \bullet \\
E & \mathcal{Y} E^{T}
\end{array}\right] \geq 0,} \\
& E^{T} \mathcal{W}=0, \quad E \mathcal{Z}^{T}=0, \\
& L_{c}(\bar{Y}, \bar{Z})+\epsilon_{1} I<0, \\
& L_{c}(\bar{X}, \bar{W})+\epsilon_{2} I<0,
\end{aligned}
$$

where

$$
\begin{aligned}
& L_{c}(\bar{Y}, \bar{Z})=\left[\begin{array}{cc}
N_{c} & 0 \\
0 & I
\end{array}\right]^{T}\left[\begin{array}{ccc}
\boldsymbol{H e}\left\{\bar{A}_{11} \bar{Y}^{T}\right\} & \bullet & \bullet \\
\bar{C}_{11} \bar{Y}^{T} & -\gamma I & \bullet \\
\bar{Z} \bar{A}_{11}^{T}+\bar{B}_{11}^{T} & D_{11}^{T}+\bar{Z} \bar{C}_{11}^{T} & -\gamma I
\end{array}\right]\left[\begin{array}{cc}
N_{c} & 0 \\
0 & I
\end{array}\right], \\
& L_{o}(\bar{X}, \bar{W})=\left[\begin{array}{cc}
N_{o} & 0 \\
0 & I
\end{array}\right]^{T}\left[\begin{array}{ccc}
\boldsymbol{H} \boldsymbol{e}\left\{\hat{A}_{22}^{T} \bar{X}\right\} & \bullet & \bullet \\
\bar{W}^{T} \hat{A}_{22}+\hat{B}_{21}^{T} \bar{X} & \boldsymbol{H e}\left\{\hat{B}_{21}^{T} \bar{W}\right\}-\gamma I & \bullet \\
\tilde{C}_{12}-C_{o} Y_{o} & D_{11} & -\gamma I
\end{array}\right]\left[\begin{array}{cc}
N_{o} & 0 \\
0 & I
\end{array}\right], \\
& N_{c}=\left[\begin{array}{c}
\bar{B}_{12} \\
D_{12}
\end{array}\right]^{\perp}, \quad N_{o}=\left[\begin{array}{ll}
\hat{B}_{22} & D_{21}
\end{array}\right]^{\perp}, \quad \bar{X}=\left[\begin{array}{ll}
0 & I_{n+n_{i}}
\end{array}\right] \mathcal{X}\left[\begin{array}{c}
0 \\
I_{n+n_{i}}
\end{array}\right] \text {, } \\
& \bar{Y}=\left[\begin{array}{ll}
I_{n+n_{o}} & 0
\end{array}\right] \mathcal{Y}\left[\begin{array}{c}
I_{n+n_{o}} \\
0
\end{array}\right], \quad \bar{W}=\left[\begin{array}{ll}
0 & I_{n+n_{i}}
\end{array}\right] \mathcal{W}, \quad \bar{Z}=\mathcal{Z}\left[\begin{array}{c}
I_{n+n_{o}} \\
0
\end{array}\right] \text {, } \\
& \tilde{C}_{12}=\left[\begin{array}{ll}
D_{o} C_{v} & \bar{C}_{11} Y_{i}+D_{12} \Pi_{i}
\end{array}\right] .
\end{aligned}
$$

Proof. Since the D-matrices of systems $\Xi_{12}$ and $\Xi_{21}$ are not full row rank and not full column rank, respectively, a reduced-order $\mathcal{H}_{\infty}$ controller exists. Then, the proof is achieved by applying the results of Xin et al. (2008) to Theorem 4.1.

To construct the reduced-order control, let $\left(\mathcal{X}_{m}, \mathcal{Y}_{m}, \mathcal{W}_{m}, \mathcal{Z}_{m}\right)$ be solutions to $\min _{\mathcal{X}, \mathcal{Y}, \mathcal{W}, \mathcal{Z}} \operatorname{Tr}\left(E^{T} \mathcal{X}+E \mathcal{Y}^{T}\right)$, subject to the conditions (8), (9), and (22)-(25). Then, there holds

$$
\mathcal{X}_{m}=\left[\begin{array}{cc}
\mathcal{X}_{m 11} & 0 \\
\mathcal{X}_{p 21} & \mathcal{X}_{p 22}
\end{array}\right], \quad \mathcal{Y}_{m}=\left[\begin{array}{cc}
\mathcal{Y}_{m 11} & \mathcal{Y}_{p 12} \\
0 & \mathcal{Y}_{p 22}
\end{array}\right]
$$

Let an SVD of $\mathcal{Y}_{m 11}-\mathcal{X}_{m 11}^{-1}$ be

$$
\mathcal{Y}_{m 11}-\mathcal{X}_{m 11}^{-1}=U \operatorname{Diag}\left(\lambda_{1}, \lambda_{2}, \cdots, \lambda_{\mu}\right) V
$$

where $U, V$ are unitary matrices, and $\lambda_{i}, i=1,2, \cdots, \mu$ are singular values of $\mathcal{Y}_{m 11}-\mathcal{X}_{m 11}^{-1}$ listed in a descending manner. Assume that there exist $\nu$ singular values negligible with respect to others. Then, we set

$$
\hat{\mathcal{Y}}_{m 11}=\mathcal{X}_{m 11}^{-1}+U \operatorname{Diag}\left(\lambda_{1}, \cdots, \lambda_{\mu-\nu}, 0 \cdots, 0\right) V .
$$

Similarly, we define matrices $\mathcal{X}_{11}$ and $\mathcal{Y}_{11}$ as

$$
\mathcal{X}_{11}=\left[\begin{array}{cc}
\mathcal{X}_{m 11} & \mathcal{X}_{p c 11} \\
\mathcal{X}_{c p 11} & \mathcal{X}_{c 11}
\end{array}\right], \quad \mathcal{X}_{11}^{-1}=\mathcal{Y}_{11}=\left[\begin{array}{cc}
\hat{\mathcal{Y}}_{m 11} & \mathcal{Y}_{p c 11} \\
\mathcal{Y}_{c p 11} & \mathcal{Y}_{c 11}
\end{array}\right]
$$




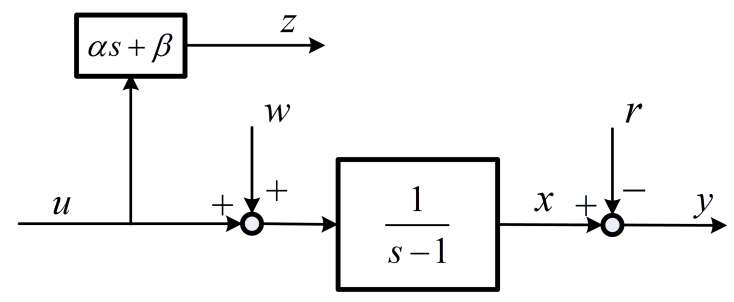

Figure 3. Example

with $\mathcal{X}_{p c 11} \mathcal{X}_{c 11}^{-1} \mathcal{X}_{c p 11}=\mathcal{X}_{m 11}-\hat{\mathcal{Y}}_{m 11}^{-1}$. Then, the underlying closed-loop Lyapunov matrix is constructed by (20). The reduced-order $\mathcal{H}_{\infty}$ controller $\mathcal{K}$ can be also obtained by solving the LMI condition (21). The following algorithm gives a way to form a reduced-order controller for solving Problem 2.1.

Algorithm 4.4 (Reduced-order Solution).

(1) Simultaneously solve the generalized Sylvester-type equations (8) and (9), and LMI conditions (14)-(17). Get solutions $\left(X_{i}, Y_{i}, \Pi_{i}, X_{o}, Y_{o}, \Pi_{o}\right)$ and $(\mathcal{X}, \mathcal{Y}, \mathcal{W}, \mathcal{Z})$

(2) Choose $\epsilon_{1}$ and $\epsilon_{2}$ such that $0<\epsilon_{1}<\lambda_{\min }\left(-L_{c}(\bar{Y}, \bar{Z})\right)$ and $0<\epsilon_{2}<$ $\lambda_{\min }\left(-L_{o}(\bar{X}, \bar{W})\right)$.

(3) Solve $\min _{\mathcal{X}, \mathcal{Y}, \mathcal{W}, \mathcal{Z}} \operatorname{Tr}\left(E^{T} \mathcal{X}+E \mathcal{Y}^{T}\right)$, subject to the conditions (22)-(25). Get solutions $\left(\mathcal{X}_{m}, \mathcal{Y}_{m}, \mathcal{W}_{m}, \mathcal{Z}_{m}\right)$.

(4) Based on $\mathcal{X}_{m}$ and $\mathcal{Y}_{m}$, form the Lyapunov matrix $\mathcal{X}_{C L}$ (20), and obtain a reducedorder $\mathcal{H}_{\infty}$ controller $\mathcal{K}$ by solving $(21)$.

(5) Obtain the controller $K$ by (10) with $\left(X_{i}, Y_{i}, \Pi_{i}, X_{o}, Y_{o}, \Pi_{o}\right)$ and $\mathcal{K}$.

\section{Simulation illustration}

In this section, we use an unstable first order system to demonstrate the effectiveness of previous results. Consider the system depicted in Fig. 3. Here, we attempt to make the state $x(t)$ follow a unit step $r(t)=1, t \geq 0$. Hence, the tracking error is defined as $e(t)=x(t)-r(t)$. Meanwhile, it is assume that $y(t)=e(t)$, that is an error feedback controller is appealed. Moreover, we penalize the control law $u(t)$ with a nonproper weight specified by $\alpha s+\beta$, where $\alpha=0.5$ and $\beta=1$. Therefore, the system data are as follows

$$
\begin{aligned}
& A_{g}=1, \quad B_{w}=B_{u}=1, \quad B_{r}=0, \quad C_{g}=1, \quad D_{r}=-1, \quad D_{w}=0, \quad C_{v}=0, \\
& D_{v r}=D_{v w}=0, \quad D_{v u}=1, \quad C_{e}=1, \quad D_{e r}=-1, \quad D_{e u}=D_{e w}=0, \quad A_{i}=0, \\
& C_{i}=1, \quad E_{o}=\left[\begin{array}{ll}
1 & 0 \\
0 & 0
\end{array}\right], \quad A_{o}=\left[\begin{array}{ll}
0 & 1 \\
1 & 0
\end{array}\right], \quad B_{o}=\left[\begin{array}{c}
0 \\
-1
\end{array}\right], \quad C_{o}=\left[\begin{array}{ll}
0 & 0.5
\end{array}\right], \quad D_{o}=1 .
\end{aligned}
$$

First we design a full-order $\mathcal{H}_{\infty}$ controller with $\gamma=1.5$. Simultaneously solving generalized Sylvester-type equations (8), (9) and the LMIs (14)-(17) yields 


$$
\begin{aligned}
& X_{i}=\left[\begin{array}{c}
-1 \\
0 \\
1
\end{array}\right], \quad Y_{i}=\left[\begin{array}{c}
1 \\
0 \\
-1
\end{array}\right], \quad \Pi_{i}=1, \quad X_{o}=\left[\begin{array}{ll}
0 & 0 \\
0 & 0
\end{array}\right], \quad Y_{o}=\left[\begin{array}{cc}
0 & 0 \\
-1 & 1
\end{array}\right] \quad \Pi_{o}=\left[\begin{array}{l}
1 \\
0
\end{array}\right], \\
& \mathcal{X}=\left[\begin{array}{cccc}
4.6340 & -6.0760 & 0.4202 & 0 \\
-6.0760 & 14.6977 & -0.4036 & 0 \\
0.4202 & -0.4036 & 6.1263 & 0 \\
0.4016 & -0.4023 & 0.4201 & 0
\end{array}\right], \quad \mathcal{W}^{T}=\left[\begin{array}{llll}
0 & 0 & 0 & 0
\end{array}\right], \\
& \mathcal{Y}=\left[\begin{array}{cccc}
4.0267 & 4.2060 & -0.3858 & 2.4954 \\
4.2060 & 6.6941 & 2.1276 & 0 \\
-0.3858 & 2.1276 & 6.0479 & -2.8476 \\
0 & 0 & 0 & -6.5825
\end{array}\right], \quad \mathcal{Z}=\left[\begin{array}{llll}
0 & 0 & 0 & -0.8696
\end{array}\right] .
\end{aligned}
$$

Then, by Algorithm 4.2, the following controller, denoted as $K_{1}$, is obtained

$$
K_{1}=\frac{1.9556(s-135.1)(s+27.64)(s+2.502)(s+0.1712)}{s(s+47.04)(s+15.19)(s+5.563)(s+2.437)} .
$$

Second, we use Algorithm 4.4 to construct a reduced-order $\mathcal{H}_{\infty}$ controller with the same level of disturbance attenuation. With $\epsilon_{1}=\epsilon_{2}=0.0008$, we obtain

$$
\begin{aligned}
\mathcal{X}_{m} & =\left[\begin{array}{cccc}
2.2907 & -2.4659 & 0.7677 & 0 \\
-2.4659 & 4.2337 & -0.9921 & 0 \\
0.7677 & -0.9921 & 1.1222 & 0 \\
0.7316 & -0.8123 & 0.9301 & 0
\end{array}\right], \quad \mathcal{W}^{T}=\left[\begin{array}{llll}
0 & 0 & 0 & 0
\end{array}\right], \\
\mathcal{Y}_{m} & =\left[\begin{array}{cccc}
1.3078 & 0.9072 & -0.0319 & 1.9593 \\
0.9072 & 1.4542 & 0.8591 & -0.1130 \\
-0.0319 & 0.8591 & 1.8491 & -3.8675 \\
0 & 0 & 0 & -3.3741
\end{array}\right], \quad \mathcal{Z}=\left[\begin{array}{llll}
0 & 0 & 0 & -0.8810
\end{array}\right] .
\end{aligned}
$$

Hence, there holds

$$
\mathcal{Y}_{m 11}-\mathcal{X}_{m 11}^{-1}=U \operatorname{diag}\left(1.5564,8.9535 \times 10^{-9}, 3.4332 \times 10^{-9}\right) V,
$$

which indicates that the last two singular values are negligible. Hence

$$
\hat{\mathcal{Y}}_{m 11}=\mathcal{X}_{m 11}^{-1}+U \operatorname{diag}(1.5564,0,0) V=\left[\begin{array}{ccc}
1.3078 & 0.9071 & -0.0319 \\
0.9072 & 1.4542 & 0.8591 \\
-0.0319 & 0.8591 & 1.8491
\end{array}\right] \text {. }
$$

Therefore, the reduced-order $\mathcal{H}_{\infty}$ controller denoted as $K_{2}$ is given as follows

$$
K_{2}=\frac{-6.1685(s+0.8958)(s+0.226)}{s(s+1.001)(s+3.005)}
$$

Finally, based on the same solutions to the generalized Sylvester-type equations, a stabilizing controller denoted as $K_{3}$ is also designed by making (11) merely stabilize 


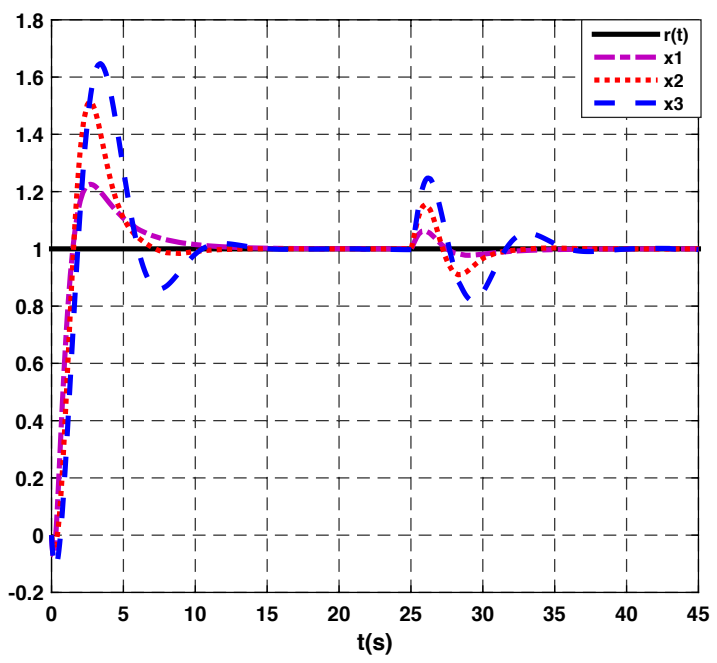

Figure 4. Asymptotic tracking with different controllers

the auxiliary system (13), and is given by

$$
K_{3}=\frac{9.3669(s-3332)(s+1.726)(s+0.4105)(s+0.1903)}{s(s+370.7)(s+66.19)(s+1.256)(s+0.4684)} .
$$

Fig. 4 exhibits the tracking performance obtained by using these three controllers. The purple curve $\left(x_{1}\right)$, the red curve $\left(x_{2}\right)$, and the blue curve $\left(x_{3}\right)$ present the state trajectories by using the full-order $\mathcal{H}_{\infty}$ controller, reduced-order $\mathcal{H}_{\infty}$ controller, and stabilizing controller, respectively. Note that all controllers contain an integrator for achieving asymptotic tracking. This specific structure is also explicitly shown in (10). Moreover, when $t=25 \mathrm{~s}$, a disturbance signal $w(t)=0.3 t e^{-2 t}$ is further added. The curve obtained with the stabilizing controller $K_{3}$ is affected much more significantly compared with the two $\mathcal{H}_{\infty}$ controllers. From this example, it is observed that the fullorder $\mathcal{H}_{\infty}$ controller leads no surprise to the best transient performance, the stabilizing controller gives relatively bad transient performance, and a tradeoff between transient performance and design complexity is reached by the reduced-order $\mathcal{H}_{\infty}$ controller.

Note that the semidefinite programming solver SeDuMi Sturm (1999) in MATLAB ${ }^{\circledR}$ is used to construct controllers, and the running times are $0.718 \mathrm{~s}, 0.863 \mathrm{~s}$, and $0.689 \mathrm{~s}$ for the full-order $H_{\infty}$ controller, reduced-order $H_{\infty}$ controller, and stabilizing controller, respectively.

\section{Conclusion}

A multiobjective control problem is addressed in this paper, where the worst-case energy gain from a finite energy disturbance to a certain performance output weighted by a nonproper filter is assumed to be bounded, together with an exact asymptotic tracking requirement. A structured controller, simultaneously achieving asymptotic tracking and stabilization under nonproper weights, is conducted by solving two generalized Sylvester-type equations. Using this parameterization, the defined multiobjective problem is transformed into a standard robust disturbance attenuation problem 
for an auxiliary system, and non conservative LMI-based solutions, for both full-order and reduced-order controllers synthesis problems, are given. One of our future research topics is to expand the current results to the case in which both the plant and the weights contain parametric dependence uncertainties.

\section{Funding}

The work was supported by the Natural Science Foundation of China under Grant 61573318 and Zhejiang Provincial Natural Science Foundation of China for Distinguished Young Scholars under Grant LR17F030003.

\section{References}

Andreev, A., \& Peregudova, O. (2017). Trajectory tracking control for robot manipulators using only position measurements. International Journal of Control, DOI: 10.1080/00207179.2017.1397755.

Cai, G., Chen, B., Peng, K., Lee, T., \& Dong, M. (2008). Comprehensive modeling and control of the yaw channel of a UAV helicopter. IEEE Transactions on Industrial Electronics, 55, 3426-3434.

Chen, B., Lee, T., Peng, K., \& Venkataramanan, V. (2003). Composite nonlinear feedback control for linear systems with input saturation: theory and an application. IEEE Transactions on Automatic Control, 48, 427-439.

Chiang, R., \& Safonov, M. G. (1992). Robust control toolbox user's guide. The Mathworks, South Natick, MA.

Dai, L. (1989). Singular control systems. Berlin: Springer-Verlag.

Fadakar, I., Fidan, B., \& Huissoon, J. (2017). Coordinate independent adaptive attitude tracking control design for spacecraft robust to time-varying system uncertainties. International Journal of Control, 90(10), 2206-2226.

Fauvel, C., Claveau, F., \& Chevrel, P. (2013). A generically well-posed $\mathrm{H}_{2}$ control problem for a one shot feedforward and feedback synthesis. In 17th international conference on system theory, control and computing (p. 201-206). Sinaia, Romania.

Feng, Y., \& Ho, D. (2014). Transient performance for discrete-time singular systems with actuators saturation via composite nonlinear feedback control. International Journal of Robust and Nonlinear Control, 24, 955-967.

Feng, Y., \& Yagoubi, M. (2016). Comprehensive admissibility for descriptor systems. Automatica, 66, 271-275.

Feng, Y., \& Yagoubi, M. (2017). Robust control of linear descriptor systems. Singapore: Springer.

Feng, Y., Yagoubi, M., \& Chevrel, P. (2012a). Extended $\mathrm{H}_{2}$ controller synthesis for continuous descriptor systems. IEEE Transactions on Automatic Control, 57(6), 1559-1564.

Feng, Y., Yagoubi, M., \& Chevrel, P. (2012b). $\mathrm{H}_{\infty}$ control with unstable and nonproper weights for descriptor systems. Automatica, 48(5), 991-994.

Francis, B. (1977). The linear multivariable regulation problem. SIAM Journal on Control and Optimization, 15, 486-505.

Gahinet, P., \& Apkarian, P. (1994). A linear matrix inequality approach to $\mathrm{H}_{\infty}$ control. International Journal of Robust and Nonlinear Control, 4(4), 421-448.

Köroğlu, H., \& Scherer, C. (2011). Robust generalized asymptotic regulation against nonstationary sinusoidal disturbances with uncertain frequencies. International Journal of Robust and Nonlinear Control, 21, 883-903.

Kwakernaak, H. (1993). Robust control and $\mathrm{H}_{\infty}$ optimization-tutorial paper. Automatica, 29, 255-273. 
Lan, W., Thum, C., \& Chen, B. (2010). A hard disk drive servo system design using composite nonlinear feedback control with optimal nonlinear gain tuning methods. IEEE Transactions on Industrial Electronics, 57, 1735-1745.

Liu, K., Zhang, H., \& Mita, T. (1997). Solution to nonsingular $\mathrm{H}_{2}$ optimal control problem with unstable weights. Systems \& Control Letters, 32, 1-10.

Masubuchi, I. (2007). Output feedback controller synthesis for descriptor systems satisfying closed-loop dissipativity. Automatica, 43, 339-345.

Meinsma, G. (1995). Unstable and nonproper weights in $\mathrm{H}_{\infty}$ control. Automatica, $31(11)$, 1655-1658.

Meng, Z., Yang, T., \& Dimarogonas, D. (2015). Coordinated output regulation of heterogeneous linear systems under switching topologies. Automatica, 53, 362-368.

Mita, T., Xin, X., \& Anderson, D. (2000). Extended $\mathrm{H}_{\infty}$ control $-\mathrm{H}_{\infty}$ control with unstable weights. Automatica, 36, 735-741.

Pipeleers, G., Demeulenaere, B., Schutter, J., \& Swevers, J. (2008). Robust high-order repetitive control: optimal performance trade-offs. Automatica, 44, 2628-2634.

Rosenbrock, H. (1974). Structural properties of linear dynamic systems. International Journal of Control, 20(2), 191-202.

Stoorvogel, A., Saberi, A., \& Sannuti, P. (2000). Performance with regulation constraints. Automatica, 36, 1443-1456.

Sturm, J. (1999). Using SeDuMi 1.02 a MATLAB toolbox for optimization over symmetric cones. Optimization Methods and Software, 11-12, 625-653.

Su, Y., \& Huang, J. (2014). Cooperative semi-global robust output regulation for a class of nonlinear uncertain multi-agent systems. IEEE Transactions on Automatic Control, 50 , 1053-1065.

Tomizuka, M. (2008). Dealing with periodic disturbances in controls of mechanical systems. Annual Reviews in Control, 32, 193-199.

Wang, H., Yung, C., \& Chang, F. (2006). H control for nonlinear descriptor systems. London: Springer-Verlag.

Wieland, P., Sepulchre, R., \& Allgöwer, F. (2011). An internal model principle is necessary and sufficient for linear output synchronization. Automatica, 47, 1068-1074.

Wonham, W. (1985). Linear multivariable control: a geometric approach (3rd ed.). New York: Springer.

Xin, X., Hara, S., \& Kaneda, M. (2008). Reduced-order proper $\mathrm{H}_{\infty}$ controllers for descriptor systems: Existence conditions and LMI-based design algorithms. IEEE Transactions on Automatic Control, 53(5), 1253-1258.

$\mathrm{Xu}$, S., \& Lam, J. (2006). Robust control and filtering of singular systems. Berlin: SpringerVerlag.

Yu, J., Zhao, Y., \& Wu, Y. (2014). Global robust output regulation control for cascaded nonlinear systems using the internal model principle. International Journal of Control, $87(4), 802-811$

Zhang, Z., \& Xie, X. (2014). Asymptotic tracking control of uncertain nonlinear systems with unknown actuator nonlinearity and unknown gain signs. International Journal of Control, 87(11), 2294-2311.

\section{Appendix A. Ddescriptor systems}

In this appendix, we recall some fundamental notations for continuous-time descriptor systems. Consider the following LTI descriptor system

$$
\left\{\begin{array}{l}
E \dot{x}(t)=A x(t)+B u(t), \\
y(t)=C x(t)+D u(t),
\end{array}\right.
$$


where $x \in \mathbb{R}^{n}, y \in \mathbb{R}^{p}$ and $u \in \mathbb{R}^{m}$ are the descriptor variable, measurement and control input vector, respectively. The matrix $E \in \mathbb{R}^{n \times n}$ may be $\operatorname{singular}$, i.e. $\operatorname{rank}(E)=r \leq$ $n$; and $A, B, C$ and $D$ are known real constant matrices with appropriate dimensions. The finite eigenvalues of $s E-A$ are called the finite dynamic modes. The infinite eigenvalues of $s E-A$ with the eigenvector $v$ satisfying $E v=0$ determine the static modes; while the infinite eigenvalues with the generalized eigenvectors $v_{k}$ such that $E v_{1}=0$ and $E v_{k}=A v_{k-1}(k \geq 2)$ are the impulsive modes.

Definition A.1. Dai (1989) The descriptor system (A1) is said to be

a) regular if $\operatorname{det}(s E-A)$ is not identically null;

b) impulse-free if $\operatorname{deg}(\operatorname{det}(s E-A))=\operatorname{rank}(E)$;

c) stable if all the roots of $\operatorname{det}(s E-A)=0$ have negative real parts;

d) admissible if it is regular, impulse-free and stable;

e) finite dynamics stabilizable (respectively finite dynamics detectable) if there exists $F$ such that $(E, A+B F)$ is regular and stable (respectively if there exists $L$ such that $(E, A+L C)$ is regular and stable);

f) impulse controllable (respectively impulse observable) if there exists $F$ such that $(E, A+B F)$ is regular and impulse-free (respectively if there exists $L$ such that $(E, A+L C)$ is regular and impulse-free).

If the descriptor system is regular, then its transfer function can be defined as

$$
T(s)=C(s E-A)^{-1} B+D .
$$

Definition A.2 (Restricted System Equivalence). Rosenbrock (1974) Consider the descriptor system (A1) and $\bar{T}$ given by

$$
\bar{T}:\left\{\begin{aligned}
\bar{E} \dot{\bar{x}}(t) & =\bar{A} \bar{x}(t)+\bar{B} \bar{u}(t) \\
\bar{y}(t) & =\bar{C} \bar{x}(t)+\bar{D} \bar{u}(t)
\end{aligned}\right.
$$

The two systems are termed restricted system equivalent, if there exist nonsingular matrices $M$ and $N$ such that

$$
\left[\begin{array}{cc}
M & 0 \\
0 & I
\end{array}\right]\left[\begin{array}{c|c}
A-s E & B \\
\hline C & D
\end{array}\right]\left[\begin{array}{cc}
N & 0 \\
0 & I
\end{array}\right]=\left[\begin{array}{c|c}
\bar{A}-s \bar{E} & \bar{B} \\
\hline C & D
\end{array}\right] .
$$

